\title{
BAT: Small and Fast KEM over NTRU Lattices
}

\author{
Pierre-Alain Fouque ${ }^{1}$, Paul Kirchner ${ }^{1}$, Thomas Pornin ${ }^{2}$ and Yang $\mathrm{Yu}^{3 \dagger}$ \\ Rennes Univ, IRISA, Rennes, France \\ pa.fouque@gmail.com, paul.kirchner@irisa.fr \\ NCC Group, Quebec, Canada \\ thomas.pornin@nccgroup.com \\ BNRist, Tsinghua University, Beijing, China \\ yang. yu0986@gmail.com
}

\begin{abstract}
We present BAT - an IND-CCA secure key encapsulation mechanism (KEM) that is based on NTRU but follows an encryption/decryption paradigm distinct from classical NTRU KEMs. It demonstrates a new approach of decrypting NTRU ciphertext since its introduction 25 years ago. Instead of introducing an artificial masking parameter $p$ to decrypt the ciphertext, we use 2 linear equations in 2 unknowns to recover the message and the error. The encryption process is therefore close to the GGH scheme. However, since the secret key is now a short basis (not a vector), we need to modify the decryption algorithm and we present a new NTRU decoder. Thanks to the improved decoder, our scheme works with a smaller modulus and yields shorter ciphertexts, smaller than RSA-4096 for 128-bit classical security with comparable public-key size and much faster than RSA or even ECC. Meanwhile, the encryption and decryption are still simple and fast in spite of the complicated key generation. Overall, our KEM has more compact parameters than all current lattice-based schemes and a practical efficiency. Moreover, due to the similar key pair structure, BAT can be of special interest in some applications using Falcon signature that is also the most compact signature in the round 3 of the NIST post-quantum cryptography standardization. However, different from Falcon, our KEM does not rely on floating-point arithmetic and can be fully implemented over the integers.
\end{abstract}

Keywords: Lattice-based cryptography · NTRU · KEM · Falcon

\section{Introduction}

Lattice-based schemes, especially when they have a polynomial structure, are a very strong contender for post-quantum cryptography. They can be faster than widely deployed cryptosystems based on RSA and ECDH. However, the sizes of public keys, signatures and ciphertexts are significantly larger than in RSA and even larger by an order of magnitude compared with ECDH cryptosystems. Such a large size is a major drawback of lattice schemes, and can be a crucial obstacle in the following situations:

- Real-world protocols may have a maximum length designed for classical cryptography. For a standard Ethernet connection, the maximum transmission unit (MTU) is 1500 bytes $^{1}$, and forward secrecy requires several objects including public keys (certificates), ciphertexts, signatures, for confidentiality and authentication.

- Large communication sizes increase the risk of lost packets and delays. Recent experiments on post-quantum TLS [PST20] show that communication sizes come

\footnotetext{
$\dagger$ Corresponding author: Yang Yu.

${ }^{1}$ https://en.wikipedia.org/wiki/Maximum_transmission_unit and Distributions on IP packets.
} 
to govern the performance when the packet loss rate is higher than 3\%. Moreover, [SKD20a] examines how the initial TCP window size affects post-quantum TLS and SSH performance, and shows that even a small size increase can reduce the observed post-quantum slowdown by $50 \%$. In addition, transmission energy can also be a significant part of the energy consumption on cryptography [Saa19]. The size of signature schemes in TLS handshakes is also important as analyzed in [SKD20b].

- In some lightweight applications, e.g. internet of things (IoT), encryption and verification are done by some constrained devices. These devices only have small on-board storage and modest processors so that they may not be compatible with large public keys, signatures and ciphertexts.

With the preparation and deployment of post-quantum cryptography underway, it is important to explore new lattice-based cryptosystems with smaller parameters.

In this light, a natural choice is NTRU [HPS98], as its structure reduces the data to one ring element. There have been many high-performance NTRU-based schemes ranging from Falcon $\left[\mathrm{FHK}^{+}\right.$20], BLISS [DDLL13] for signature to NTRU-HRSS [HRSS17], NTTRU [LS19], NTRUEncrypt [CHWZ17], NTRU Prime [BCLvV17] for encryption and KEM. In particular, Falcon is the most compact signature in the round 3 of the NIST post-quantum cryptography standardization [NIS20]. In addition, NTRU-based encryption and KEM schemes have some advantage in real-world use: the relevant patents have expired; by contrast, there could be some (controversial) intellectual property claims on the Ring/Module-LWE counterparts.

NTRU-based schemes are defined over some polynomial ring $\mathcal{R}$ that is $\mathcal{R}=\mathbb{Z}[x] /\left(x^{n}+1\right)$ with $n$ a power-of- 2 in this work. The secret key of an NTRU cryptosystem is essentially a pair of short polynomials $(f, g) \in \mathcal{R}^{2}$ while the public key is $h=f^{-1} g \bmod q$. All NTRU encryption schemes, ranging from the earliest proposal [HPS98] to the round 3 NIST submission $\left[\mathrm{CDH}^{+} 20\right]$, have followed essentially the same design rationale for more than 20 years. Concretely, the ciphertext is $c=p h r+m \bmod q$ where $p$ is the masking modulus, $r$ is the randomness, $m$ is the message. A correct decryption is built upon that $c^{\prime}=p g r+f m$ is short so that $c^{\prime}=(f c \bmod q)$. The masking modulus $p$ is also necessary to decrypt: one needs to first clean out $p g r$ via reduction modulo $p$ and then to recover $m$ by multiplying the inverse of $f$ modulo $p$. For typical NTRU KEMs, $(f, g)$ is sparse and of length about $C \sqrt{n}$ for small constant $C$.

By contrast, the design rationale for NTRU-based signatures went through some significant changes. The first NTRU-based signature is NTRUSign $\left[\mathrm{HHP}^{+} 03\right]$ that is a hash-andsign scheme. However, its signature transcripts leak some secret key information so that NTRUSign and some variants were broken by statistical attacks [NR06, DN12]. Later, Ducas et al. made use of the GPV hash-and-sign framework [GPV08] and proposed a provably secure NTRU-based signature [DLP14] that further developed into Falcon [FHK $\left.{ }^{+} 20\right]$. The public key of Falcon is still $h=f^{-1} g \bmod q$ for some short $(f, g)$, while the actual secret key is a trapdoor basis $\mathbf{B}_{f, g}=\left(\begin{array}{ll}g & G \\ f & F\end{array}\right) \in \mathcal{R}^{2 \times 2}$ such that $g F-f G=q$. The signing of Falcon is essentially Gaussian sampling with a trapdoor [GPV08, DP16]. Consequently, the signature size depends on the maximal Gram-Schmidt norm of $\mathbf{B}_{f, g}$. As analyzed in [DLP14], Falcon chooses $(f, g)$ of $\|(f, g)\| \approx 1.17 \sqrt{q}$ for optimal parameters. Therefore, $\|(f, g)\|$ in Falcon is independent of $n$, which is different from the case of NTRU KEMs.

Some other NTRU-based signatures [DDLL13, $\left.\mathrm{DHP}^{+} 20\right]$ indeed use one vector $(f, g)$ as the secret key as the case of NTRU KEMs. However, to the best of our knowledge, there is no practical NTRU-based KEM using a trapdoor basis as the secret key. Intuitively we expect $(F, G)$ to yield one more equation in decryption so that one can recover both the message and encryption randomness via two equations. This in effect gets rid of the masking modulus $p$ in classical NTRU KEMs and thus hopefully allows smaller parameters. It is noteworthy that the improvement in compactness of NTRU-based KEMs severely lags 
that in speed of NTRU-based KEMs [LS19, DHK $\left.{ }^{+} 21\right]$ and in compactness of LWE-based KEMs $\left[\mathrm{BMD}^{+} 20, \mathrm{LLJ}^{+} 19, \mathrm{BBC}^{+} 20, \mathrm{CKLS18}\right]$. Moreover, when we unify the trapdoor function for both signature and KEM, part of the code can be shared and we may also reduce some storage and communication. Therefore, it would be interesting to investigate the practicality of a trapdoor basis for KEM.

Indeed, the earliest lattice-based cryptosystem GGH (Goldreich-Goldwasser-Halevi [GGH97a]) uses a trapdoor basis as the secret key and implements both encryption and signature based on that trapdoor function. Later Micciancio improved the GGH trapdoor function by using the Hermite normal form [Mic01]. While GGH encryption has a long history as NTRU, its practicality is far from well-studied and there is no GGH-like encryption/KEM with concrete parameter and security analysis so far.

Our contributions. We present a new KEM based on NTRU, called BAT. ${ }^{2}$ Similar to Falcon signature, BAT uses $h=f^{-1} g \bmod q$ as the public key and its secret key is a trapdoor basis $\mathbf{B}_{f, g}$ with an additional ring element (for faster decapsulation). In addition, BAT shares the same leading design principle with Falcon, i.e. minimizing the communication size.

Our main improvement in BAT-KEM is a better decapsulation algorithm, which represents a major modification to the NTRU encryption scheme since its introduction 25 years ago. Instead of following the original NTRU, we modify it according to the GGH-Micciancio blueprint [GGH97b, Mic01]. The message $m$ is now encapsulated as $c=h m+e \bmod q$ where $h$ is the NTRU public key and $e$ is a small error. The decapsulation corresponds to applying Babai's nearest plane algorithm with the secret basis to decode the closest lattice point, and therefore recovering the message. Compared with other NTRU KEMs, we do not need the masking modulus $p$ to extract the message. Instead of multiplying only by $f$, we multiply by $F$ so that we get 2 linear equations in the 2 unknowns $e, m$.

However, Babai's nearest plane algorithm heavily relies on floating-point arithmetic, although most expensive calculation can be done in a pre-computation phase. To avoid floating-point arithmetic in the decapsulation, we replace the high-precision Gram-Schmidt vectors with integral approximations. Additionally, notice that $m$ and $e$ do not necessarily follow the identical distribution, hence we take into account their different sizes to optimize the decoding. We also use the Learning With Rounding (LWR) assumption [BPR12, CZZ18] in order to further reduce the size of the ciphertext. Our improved decoding algorithm can be used with a smaller modulus and dropping more bits, and thereby increases the security of the scheme and decreases the communication.

Overall, our KEM achieves very impressive performance. First of all, for the same NIST security level, BAT achieves the smallest communication size, namely "public key size + ciphertext size", among all current lattice cryptosystems and even RSA cryptosystems. Secondly, the complexity of the code as well as its running time is asymmetric: while the key generation is complicated, the frequent key usage is quite efficient. Specifically, the encryption is very simple - essentially a ring multiplication —, and the decryption also boils down to a few ring additions and multiplications. Cheap daily operations make BAT particularly compatible with small devices. Thirdly, we can implement the whole scheme fully over integers, which is different from the case of Falcon. Our implementation is constant-time and uses some AVX2 optimizations. We can notice that BAT has performance comparable to Kyber while being more compact. Furthermore, it is comparable to SIKE p434 in size while being much more efficient. We gave the timing with $\mathrm{x} 86$ assembly optimization, while with the same level of optimization we did, the SIKE performance timing would be higher by one order of magnitude. We summarize the detailed comparisons with some well-known schemes in Table 2.

\footnotetext{
${ }^{2}$ BAT stands for "Basis with Attractive Trapdoor".
} 
Finally, we explain in a simplistic way why the new decryption algorithm leads to smaller parameters. To correctly decrypt, our KEM needs $(f c \bmod q)=g m+f e$, i.e. $\|g m+f e\|_{\infty}<\frac{q}{2}$, while previous NTRU KEMs need $\|p g r+f m\|_{\infty}<\frac{q}{2}$. We also compare with ring-LWE-based KEMs. For a typical ring-LWE-based KEM, its secret key is $(f, g) \in \mathcal{R}^{2}$, public key is $(a, b=a f+g) \in(\mathcal{R} / q \mathcal{R})^{2}$ and ciphertext is $\left(c_{1}=\right.$ $\left.a e_{0}+e_{1}, c_{2}=b e_{0}+e_{2}+\left\lfloor\frac{q}{2}\right\rceil m\right) \in(\mathcal{R} / q \mathcal{R})^{2}$. The requirement for correct decryption is $\left\|e_{0} g-e_{1} f+e_{2}\right\|_{\infty}<\frac{q}{4}$. Suppose that $m, e, r, e_{i}$ are drawn from a distribution of standard deviation $\sigma_{e}$ and $f, g$ from a distribution of standard deviation $\sigma_{f}$. The coefficients of $g m+f e, p g r+f m, e_{0} g-e_{1} f+e_{2}$ are modeled as Gaussian. The comparison on parameter restrictions are summarized in Table 1 . It can be seen that given $\left(n, \tau, \sigma_{e}, \sigma_{f}\right)$, BAT allows a smaller modulus $q$. Note that for fixed $\left(n, \sigma_{e}, \sigma_{f}\right)$, a smaller $q$ implies higher security.

Table 1: The parameter restrictions for correct decryption. The parameter $\tau$ is the tail-bound parameter determining the decryption failure rate.

\begin{tabular}{|c|c|}
\hline & Requirement for correct decryption \\
\hline NTRU & $\tau \sigma_{e} \sigma_{f} \sqrt{\left(p^{2}+1\right) n}<q / 2$ \\
Ring-LWE & $\tau \sigma_{e} \sigma_{f} \sqrt{2 n}<q / 4$ \\
BAT & $\tau \sigma_{e} \sigma_{f} \sqrt{2 n}<q / 2$ \\
\hline
\end{tabular}

Comparison with Falcon. BAT is similar in spirit to Falcon signature: they both achieve good compactness by using some nice NTRU trapdoor basis as the secret key. Nevertheless, some crucial distinctions exist between BAT and Falcon.

- At a high level, BAT and Falcon exploit their trapdoor to solve CVP (closest vector problem), but the used CVP algorithms are very different. Specifically, Falcon makes use of the KGPV Gaussian sampler [GPV08] that is a randomized Babai's nearest plane algorithm. In contrast, BAT decrypts with a deterministic NTRU decoder that can be viewed as a hybrid of Babai's round-off and nearest plane algorithms.

- The algorithms of BAT are simpler than those of Falcon. On the one hand, the signing of Falcon relies on high-precision Gaussian sampling, but the encryption and decryption of BAT only need basic integer operations. On the other hand, Falcon includes many high-precision intermediate values along with the trapdoor for faster signing, but BAT just adds one integral polynomial for faster decryption.

- The NTRU trapdoors of BAT and Falcon are generated in different ways. In fact, Falcon chooses its trapdoor for smaller signatures, which is equivalent to minimizing the maximal Gram-Schmidt norm of the trapdoor basis. As for BAT, the trapdoor is generated to minimize the decryption failure, and according to our new decoder, the distributions of the message and error will also affect the trapdoor generation (see Section 3 for more details).

Related works. Chuengsatiansup et al. $\left[\mathrm{CPS}^{+} 20\right]$ propose some extensions of Falcon signature and NTRU encryption over Module-NTRU lattices. This allows more flexible parameters for NTRU-based cryptosystems. Our techniques are likely to apply to the Module-NTRU-based schemes as well.

In recent years, the performance of NTRU encryption has been greatly improved [LS19, $\left.\mathrm{DHK}^{+} 21\right]$. These newly NTRU instantiations are mainly proposed for high efficiency and follow the classical design of original NTRU. In contrast, BAT is proposed driven by the quest for compactness and its design is different.

In order to improve parameters, some schemes [CKLS18, $\left.\mathrm{ZYF}^{+} 20\right]$ are built upon a variant of LWE in which the secret and error follow different distributions. Our work 
Table 2: Comparisons with other KEMs including NTRU-HRSS [HRSS17], NTTRU [LS19], Kyber $\left[\mathrm{ABD}^{+} 20\right]$, Saber $\left[\mathrm{BMD}^{+} 20\right]$, LAC $\left[\mathrm{LLJ}^{+} 19\right]$, Round5 [BBC $\left.{ }^{+} 20\right]$, ECC, RSA and SIKE $\left[\mathrm{JAC}^{+} 20\right]$. Timings do not include generation of a random seed (from the operating system's RNG) or key derivation costs. Sizes for BAT and LW-BAT include an optional one-byte identifying header. The implementation of LW-BAT was not fully optimized with AVX2 opcodes. Measurements for Kyber, Saber and RSA-4096 were performed on the exact same system (x86 Coffee Lake) as BAT and LW-BAT. Measurements for NTRU-HRSS and NTTRU were given in [LS19], those for LAC, Round5 and SIKE were as in their NIST documentations. Values for ECC are for the curve25519 implementation in eBACS [BL].

\begin{tabular}{|c||c|c|c|c|c|c|}
\hline & $\begin{array}{c}\text { Security } \\
\text { Level }\end{array}$ & $\begin{array}{c}\text { Ciphertext } \\
\text { (bytes) }\end{array}$ & $\begin{array}{c}\text { PK } \\
\text { (bytes) }\end{array}$ & $\begin{array}{c}\text { Keygen } \\
\text { (kcycles) }\end{array}$ & $\begin{array}{c}\text { Encaps } \\
\text { (kcycles) }\end{array}$ & $\begin{array}{c}\text { Decaps } \\
\text { (kcycles) }\end{array}$ \\
\hline BAT & NIST-I & 473 & 521 & $29.4 \times 10^{3}$ & 11.1 & 59.7 \\
BAT & NIST-V & 1006 & 1230 & $145.6 \times 10^{3}$ & 25.6 & 131.16 \\
LW-BAT & 80 bits (C) & 203 & 225 & $19.0 \times 10^{3}$ & 58.3 & 240.8 \\
\hline NTRU-HRSS & NIST-I & 1140 & 1140 & 220.3 & 34.6 & 65.0 \\
NTTRU & NIST-I & 1248 & 1248 & 6.4 & 6.1 & 7.8 \\
Kyber & NIST-I & 768 & 800 & 23.6 & 36.8 & 28.5 \\
Kyber & NIST-V & 1568 & 1568 & 57.1 & 79.8 & 64.6 \\
Saber & NIST-I & 736 & 672 & 50.0 & 59.0 & 57.2 \\
Saber & NIST-V & 1472 & 1312 & 123.6 & 141.8 & 141.0 \\
LAC & NIST-I & 712 & 544 & 59.6 & 89.1 & 140.2 \\
LAC & NIST-V & 1424 & 1056 & 135.8 & 208.0 & 359.2 \\
Round5 & NIST-I & 620 & 461 & 46 & 68 & 95 \\
Round5 & NIST-V & 1285 & 978 & 105 & 166 & 247 \\
Round5-iot & 96 bits (C) & 394 & 342 & 41 & 52 & 28 \\
\hline RSA 4096 & 128 bits (C) & 512 & 512 & $2.19 \times 10^{6}$ & 212.1 & 13690 \\
ECC & 128 bits (C) & 32 & 32 & 46 & 136 & 136 \\
SIKE p434 & NIST-I & 346 & 330 & $5.9 \times 10^{3}$ & $9.7 \times 10^{3}$ & $10.3 \times 10^{3}$ \\
Compressed & NIST-I & 236 & 197 & $10.2 \times 10^{3}$ & $15.1 \times 10^{3}$ & $11.1 \times 10^{3}$ \\
SIKE p434 & & & & & \\
\hline
\end{tabular}

${ }^{a}$ Here "xxx bits (C)" represents xxx bits of security in classical setting, which does not match any suggested NIST security level.

${ }^{\mathrm{b}}$ Round5-iot is only IND-CPA secure rather than IND-CCA secure.

${ }^{\mathrm{c}}$ SIKE and compressed-SIKE use x64 assembly optimizations.

makes use of a similar idea. Yet the main difference is that our KEM follows a novel pattern which is essential to minimize the parameters.

\section{Preliminaries}

\subsection{Notations}

We follow the setting $\mathbb{Z}_{q}=\{-(\lceil q / 2\rceil-1),-(\lceil q / 2\rceil-2), \cdots, q-\lceil q / 2\rceil\}$ and $(a \bmod q) \in \mathbb{Z}_{q}$ for any $a \in \mathbb{Z}$. Let $\ln$ (resp. log) denote the logarithm with base $e$ (resp. 2). For an integer $q>0$, let $\lfloor a\rceil_{q}=\lfloor a q\rceil / q \in(1 / q) \cdot \mathbb{Z}$ for $a \in \mathbb{R}$. For a real-valued function $f$ and a countable set $S$, we write $f(S)=\sum_{x \in S} f(x)$ assuming that this sum is absolutely convergent.

\subsection{Linear algebra}

Let $\mathbf{B}=\left(\mathbf{b}_{0}, \ldots, \mathbf{b}_{n-1}\right) \in \mathbb{Q}^{n \times n}$ of rank $n$. The Gram-Schmidt orthogonalization of $\mathbf{B}$ is $\mathbf{B}=\mathbf{B}^{*} \mathbf{U}$, where $\mathbf{U} \in \mathbb{Q}^{n \times n}$ is upper-triangular with 1 on its diagonal and $\mathbf{B}^{*}=$ 
$\left(\mathbf{b}_{0}^{*}, \ldots, \mathbf{b}_{n-1}^{*}\right)$ is a matrix with pairwise orthogonal columns.

Let $\mathcal{R}_{n}=\mathbb{Z}[x] /\left(x^{n}+1\right)$ with $n$ a power-of- 2 and $\mathbb{K}_{n}=\mathbb{Q}[x] /\left(x^{n}+1\right)$. We denote by $\left(\mathcal{R}_{n} \bmod q\right)$ the ring $\mathcal{R}_{n} / q \mathcal{R}_{n}$. When the context is clear, we may write $\mathcal{R}_{n}$ (resp. $\mathbb{K}_{n}$ ) as $\mathcal{R}$ (resp. $\mathbb{K}$ ). We identify $f=\sum_{i=0}^{n-1} f_{i} x^{i} \in \mathbb{K}_{n}$ with its coefficient vector $\operatorname{coef}(f)=\left(f_{0}, \cdots, f_{n-1}\right)$. Let $\|f\|$ (resp. $\|f\|_{\infty}$ ) denote the $\ell_{2}$-norm (resp. $\ell_{\infty}$-norm) of $\operatorname{coef}(f)$. We denote by $\bar{f}$ the conjugate of $f$, i.e. $f\left(x^{-1}\right)$. Let $\mathbf{1}=\sum_{i=0}^{n-1} x^{i} \in \mathcal{R}$. The symbol $[\cdot]_{q}$ is naturally generalized to $\mathbb{K}_{n}$ by applying it coefficient-wise.

\subsection{Probability and statistics}

Given a distribution $\chi$, we write $z \leftarrow \chi$ when the random variable $z$ is drawn from $\chi$. For $z \leftarrow \chi$, let $\mu[z]$ (resp. $\sigma[z]$ ) denote the expectation (resp. standard deviation) of $z$, and $\mu[\chi]:=\mu[z]$ (resp. $\sigma[\chi]:=\sigma[z]$ ). If $\mu[\chi]=0$, then $\chi$ is called centered. For a random $a \in \mathbb{K}$, if all its coefficients independently follow a distribution $\chi$, then we call a iid-random over $\chi$. If $a \in \mathbb{K}$ is iid-random over $\chi$, we write $\sigma_{a}=\sigma[\chi]$ and $\mu_{a}=\mu[\chi]$. We call it centered when $\mu_{a}=0$.

For a distribution $\chi$, we denote by $\operatorname{Sample}(\chi)$ the procedure of generating a random sample of $\chi$ and by Sample $(\chi$; seed) the sampling procedure with seed seed. For a finite set $S$, let $U(S)$ be the uniform distribution over $S$. In particular, for a positive integer $k$, $\sigma\left[U\left(\mathbb{Z}_{k}\right)\right]=\sqrt{\frac{k^{2}-1}{12}}$ and $\mu\left[U\left(\mathbb{Z}_{k}\right)\right]=0$ if $k$ is odd; otherwise $\mu\left[U\left(\mathbb{Z}_{k}\right)\right]=\frac{1}{2}$.

For $\sigma>0$, let $\rho_{\sigma}(x)=\exp \left(-\frac{x^{2}}{2 \sigma^{2}}\right)$ be the one-dimensional Gaussian function with standard deviation $\sigma$. The centered discrete Gaussian over integers with standard deviation $\sigma$ is defined by the probability function $D_{\mathbb{Z}, \sigma}(x)=\frac{\rho_{\sigma}(x)}{\rho_{\sigma}(\mathbb{Z})}$ for any $x \in \mathbb{Z}$. Let $\operatorname{erf}(x)=$ $\frac{2}{\sqrt{\pi}} \int_{0}^{x} \exp \left(-t^{2}\right) \mathrm{d} t$ be the error function. For a random variable $X$ following a normal distribution with mean 0 and variance $1 / 2, \operatorname{erf}(x)$ is the probability of $X$ in the range $[-x, x]$.

\subsection{NTRU}

Given $f, g \in \mathcal{R}$ such that $f$ is invertible modulo some $q \in \mathbb{Z}$, let $h=f^{-1} g \bmod q$. The NTRU lattice defined by $h$ is denoted by $\mathcal{L}_{h, q}=\left\{(u, v)^{t} \mid u=h v \bmod q\right\}$. Given $(f, g)$, one can compute $\left(\begin{array}{ll}g & G \\ f & F\end{array}\right) \in \mathcal{R}^{2 \times 2}$ a basis of $\mathcal{L}_{h, q}$ by solving the NTRU equation $g F-f G=$ $q\left[\mathrm{HHP}^{+} 03, \mathrm{PP} 19\right]$. Fixing $(f, g)$, there are infinitely many such bases, whereas these bases have the same Gram-Schmidt norms. Hence, we simply write $\mathbf{B}_{f, g}=\left(\begin{array}{ll}g & G \\ f & F\end{array}\right) \in \mathcal{R}^{2 \times 2}$.

While the public key of an NTRU-based scheme is $h$ itself, the secret key can have different forms. For most NTRU encryption schemes, the secret key is $(g, f)$ itself, i.e. one short vector of $\mathcal{L}_{h, q}$. For some other applications, e.g. signature and IBE, the secret key is $\mathbf{B}_{f, g}$ called an NTRU trapdoor basis. Falcon $\left[\mathrm{FHK}^{+} 20\right]$ is a representative example. Falcon is an NTRU-based signature following the GPV hash-and-sign framework [GPV08]. To sign a message $m$, the signer computes a pair of short polynomials $\left(s_{1}, s_{2}\right)$ such that $s_{1}+h s_{2}=\operatorname{Hash}(m)$. This procedure is accomplished by lattice Gaussian sampling with $\mathbf{B}_{f, g}$ and the length of the signature $\left(s_{1}, s_{2}\right)$ depends on the sampled Gaussian width. The Gaussian sampler of Falcon is a fast Fourier variant [DP16] of the KGPV sampler [GPV08], hence the signature size is proportional to the maximal Gram-Schmidt norm of $\mathbf{B}_{f, g}$. For optimal parameters, Falcon generates $(f, g)$ such that $\|(f, g)\| \approx 1.17 \sqrt{q}$ as per [DLP14]. 


\section{A New NTRU Decoder}

In this section we present a new NTRU decoding algorithm that is the key component of our KEM. In the context of NTRU, the code words are $h s+e \bmod q$ where $h$ is the public key and $s, e$ are small polynomials. The decoding process recovers $(s, e)$ with an NTRU trapdoor. An ideal decoder is supposed to satisfy:

1. All operations are simple and efficient; no high-precision arithmetic is needed.

2. The decoding distance is large, i.e. being able to recover large errors $(s, e)$. Note that for our KEM, larger errors correspond to higher security level.

There have been two famed decoding algorithms due to Babai [Bab86]: Babai's roundoff algorithm (RO for short) and Babai's nearest plane algorithm (NP for short). They have respective pros and cons. The RO algorithm outperforms NP in efficiency and simplicity. In addition, RO is particularly compatible with $q$-ary lattices: all operations are over $\mathbb{Z}_{q}$. By contrast, NP is capable of decoding larger errors in both the worst and average cases [Pre15]. Yet the principal drawback of NP is its reliance on high-precision arithmetic.

Our decoder overcomes the main shortcomings of RO and NP. First, it is able to tackle a larger decoding distance than RO. Second, while complicated computations are still required, all involved algorithms can be implemented using fixed-point arithmetic in practice, which outperforms NP. Meanwhile these expensive computations can be done in the pre-computation and therefore do not affect the decoding efficiency. With an auxiliary integer vector, our algorithm achieves the same efficiency as RO and all operations are integer arithmetic. To optimize the decoding, our algorithm also takes into account the distributions of $s$ and $e$.

\subsection{Babai's algorithms for NTRU}

For better contrast, we first recall RO and NP briefly in the NTRU setting. Let $h \in$ $(\mathcal{R} \bmod q)$ be the public key and $\mathbf{B}_{f, g}=\left(\begin{array}{cc}g & G \\ f & F\end{array}\right) \in \mathcal{R}^{2 \times 2}$ be the trapdoor basis. In later discussion, we shall treat $\mathcal{L}_{h, q}$ as a $\mathcal{R}$-module of rank 2 rather than a $\mathbb{Z}$-module of rank $2 n$.

The application of RO related to NTRU dates back to NTRUSign $\left[\mathrm{HHP}^{+} 03\right]$. Given $c=h s+e \in(\mathcal{R} \bmod q)$, we have $\left(\begin{array}{l}c \\ 0\end{array}\right) \in \mathcal{L}_{h, q}+\left(\begin{array}{c}e \\ -s\end{array}\right)$. The RO algorithm computes $\left(\begin{array}{c}e^{\prime} \\ -s^{\prime}\end{array}\right)=\left(\begin{array}{l}c \\ 0\end{array}\right)-\mathbf{B}_{f, g}\left\lfloor\mathbf{B}_{f, g}^{-1}\left(\begin{array}{l}c \\ 0\end{array}\right)\right]$ as $\left(\begin{array}{c}e \\ -s\end{array}\right)$. As $\mathbf{B}_{f, g}^{-1}=\frac{1}{q}\left(\begin{array}{cc}F & -G \\ -f & g\end{array}\right)$, a correct decoding such that $\left(e^{\prime}, s^{\prime}\right)=(e, s)$ follows if

$$
\left(\begin{array}{cc}
F & -G \\
-f & g
\end{array}\right)\left(\begin{array}{c}
e \\
-s
\end{array}\right)=\left(\begin{array}{c}
F c \bmod q \\
-f c \bmod q
\end{array}\right) .
$$

This is equivalent to $\max \left\{\|f e+g s\|_{\infty},\|F e+G s\|_{\infty}\right\} \leq \frac{q}{2}$.

The NP algorithm is more complicated. It involves the Gram-Schmidt orthogonal basis $\mathbf{B}_{f, g}^{*}=\left(\begin{array}{ll}g & G^{*}=G-v g \\ f & F^{*}=F-v f\end{array}\right)$ where $v=\frac{F \bar{f}+G \bar{g}}{f \bar{f}+g \bar{g}}$. A correct decoding follows if $\max \left\{\|f e+g s\|_{\infty},\left\|F^{*} e+G^{*} s\right\|_{\infty}\right\} \leq \frac{q}{2}$.

In some applications [DLP14, $\left.\mathrm{FHK}^{+} 20\right]$, the trapdoor basis is optimal with respect to Gram-Schmidt norms: $\|(g, f)\| \approx\left\|\left(G^{*}, F^{*}\right)\right\|$. However, $\|(g, f)\|$ and $\|(G, F)\|$ are not so close: $\|(G, F)\| \approx \sqrt{\frac{n}{12}} \cdot\|(g, f)\|\left[\mathrm{HHP}^{+} 03\right]$. As a consequence, $\|(e, s)\|$ is dominated by the large $\|(G, F)\|$ in the RO algorithm but by the small $\|(g, f)\|$ in the NP algorithm, which leads to a gap of $O(\sqrt{n})$. 


\subsection{Our decoding algorithm for NTRU}

As shown in Section 3.1, RO boils down to solving two linear equations over $\mathcal{R}$ (without modular reduction). To enlarge its decoding range, we hope to replace the large $(G, F)$ with some small vector $\left(G^{\prime}, F^{\prime}\right)$ of size $\approx\|(g, f)\|$. A natural candidate is $\left(G^{*}, F^{*}\right)=$ $(G-v g, F-v f)$ with $v=\frac{F \bar{f}+G \bar{g}}{f \bar{f}+g \bar{g}}$ as in NP. However, if we want to work with $\left(G^{*}, F^{*}\right)$ directly, we have to resort to high-precision arithmetic. To overcome the precision issue, we choose $\left(G^{\prime}, F^{\prime}\right)=\left(G-g\lfloor v\rceil_{q^{\prime}}, F-f\lfloor v\rceil_{q^{\prime}}\right) \in\left(1 / q^{\prime}\right) \mathcal{R}^{2}$. When $q^{\prime}$ is sufficiently large, $\left(G^{\prime}, F^{\prime}\right)$ converges to $\left(G^{*}, F^{*}\right)$ whose norm is about $\|(g, f)\|$. In practice, a moderate $q^{\prime}$ suffices to significantly improve the decoding.

We further refine our decoder as per the distributions of $s$ and $e$. We focus on the common case where both $s$ and $e$ are iid-random over some publicly known distributions $\chi_{s}$ and $\chi_{e}$. In practice, $\chi_{s}$ and $\chi_{e}$ are not necessarily the same or even close, which may cause a gap between the sizes of $s$ and $e$. For example, when $\|s\| \ll\|e\|$, we expect a better decoding by using a basis $\left(\begin{array}{ll}g^{\prime} & G^{\prime} \\ f^{\prime} & F^{\prime}\end{array}\right)$ with $\left\|g^{\prime}\right\|>\|g\| \approx\|f\|>\left\|f^{\prime}\right\|$ and $\left\|G^{\prime}\right\|>\|G\| \approx\|F\|>\left\|F^{\prime}\right\|$. To this end, we introduce a parameter $\gamma$, by default $\gamma=\sigma_{e} / \sigma_{s}$, to compute the optimal decoding basis. Moreover, $\chi_{s}$ and $\chi_{e}$ do not have to be centered, e.g. $\chi_{s}=U\left(\mathbb{Z}_{2}\right)$. For given $(f, g)$, non-centered $s$ and $e$ lead to a non-zero average of $f e+g s$. Therefore, we also consider the impact of $\mu_{s}$ and $\mu_{e}$ during decoding. Here we assume $\mu_{s}, \mu_{e} \in \frac{1}{Q} \cdot \mathbb{Z}$ for some $Q \in \mathbb{N}$, which is indeed the case of our later schemes.

The decoding algorithm consists of two steps: (1) computing the auxiliary polynomial $w$ and (2) recovering $(s, e)$. They are illustrated in Algorithms 3.1 and 3.2 respectively. Notably, both algorithms can be fully implemented over the integers. In Algorithm 3.1, the computation of $v$ consists of one polynomial division, but the final output is actually an integral approximation of $q^{\prime} v$, which can be computed with fixed-point values. More details are presented in Section 6.1.
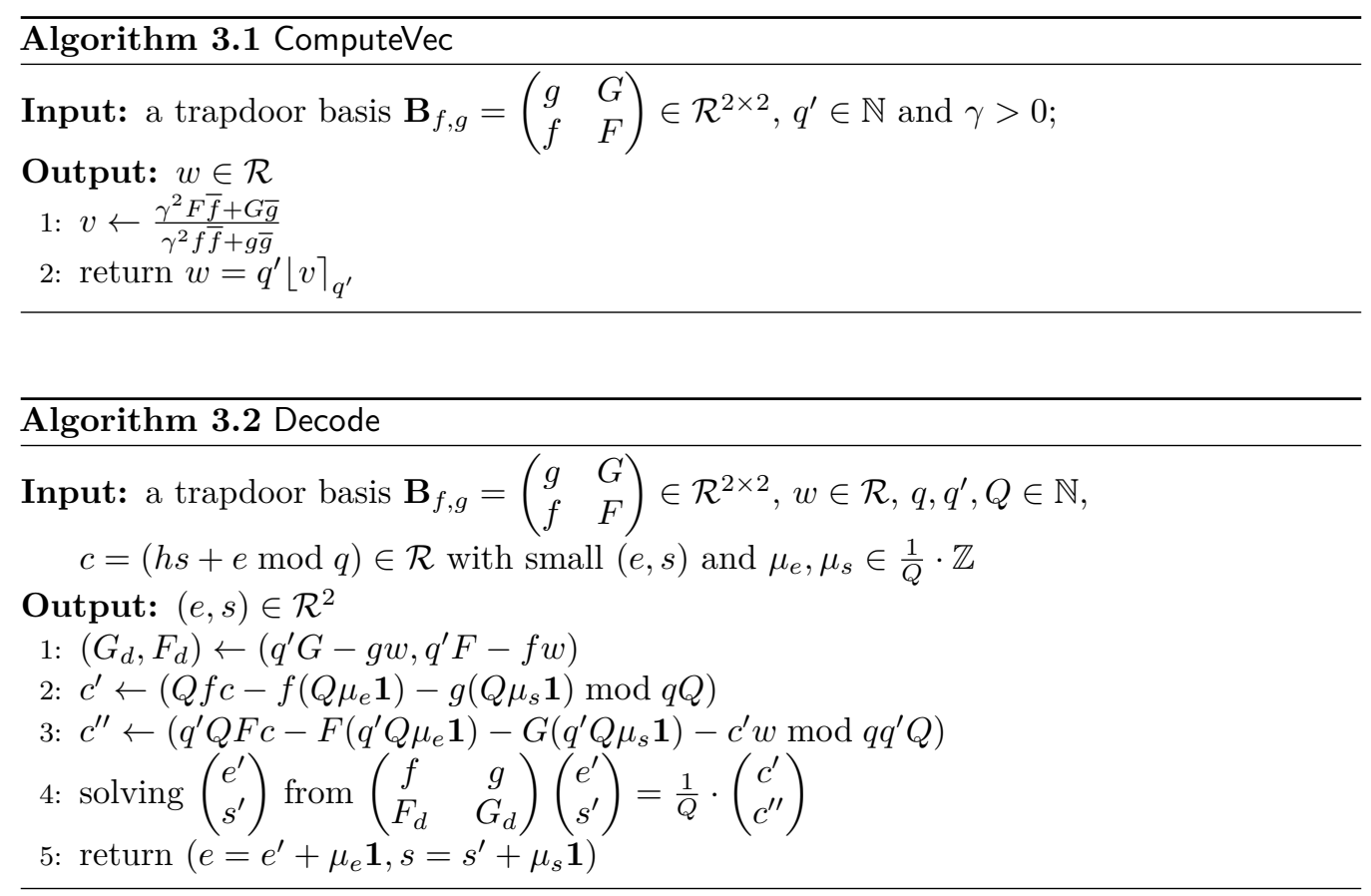

Claim 1 gives a heuristic estimation of the probability of correct decoding of Algorithm 3.2. The argument is in Appendix A. 
Claim 1. Let $\mathcal{R}=\mathbb{Z}[x] /\left(x^{n}+1\right)$ with $n$ a power-of-2 and $q \in \mathbb{N}$. Let $f, g \in \mathcal{R}$ be iidrandom over $D_{\mathbb{Z}, \sigma_{f}}$ and $h=f^{-1} g \bmod q$. Let $\mathbf{B}_{f, g}=\left(\begin{array}{cc}g & G \\ f & F\end{array}\right) \in \mathcal{R}^{2 \times 2}$ be an NTRU basis. Let $s, e \in \mathcal{R}$ be iid-random over $\chi_{s}$ and $\chi_{e}$ respectively, and $\mu_{e}, \mu_{s} \in \frac{1}{Q} \cdot \mathbb{Z}$ for some $Q \in \mathbb{N}$. Let $\gamma=\sigma_{e} / \sigma_{s}, q^{\prime} \in \mathbb{N}$ and $w=\operatorname{ComputeVec}\left(\mathbf{B}_{f, g}, q^{\prime}, \gamma\right)$. Let $I(\gamma)=\frac{2 \ln (\gamma)}{\gamma^{2}-1}$ for $\gamma \neq 1$ and $I(1)=1$. Let $\sigma_{1}=\sqrt{n} \sigma_{f} \sigma_{s} \sqrt{\gamma^{2}+1}, \sigma_{2}=\sigma_{s}\left(\frac{q \gamma}{\sqrt{n} \cdot \sigma_{f}} \sqrt{I(\gamma)}+\frac{n^{1.5} \sqrt{\gamma^{2}+1}}{2 q^{\prime}} \sigma_{f}\right)$. Let $\tau=\min \left\{\frac{q}{2 \sqrt{2} \sigma_{1}}, \frac{q}{2 \sqrt{2} \sigma_{2}}\right\}$. Then the probability of Decode $\left(\mathbf{B}_{f, g}, w, q, q^{\prime}, Q,(h s+e \bmod q)\right)=$ $(e, s)$ is heuristically estimated at least $1-2 n \cdot(1-\operatorname{erf}(\tau))$ over the randomness of $s$ and $e$.

\subsection{Decoding failure rate}

Claim 1 gives a heuristic estimate for $\mu\left[P\left(\mathbf{B}_{f, g}, w, \chi_{s}, \chi_{e}\right)\right]$ (over the randomness of $(f, g)$ ) where

$P\left(\mathbf{B}_{f, g}, w, \chi_{s}, \chi_{e}\right)=\operatorname{Pr}\left[\operatorname{Decode}\left(\mathbf{B}_{f, g}, w, q, q^{\prime}, Q,(h s+e \bmod q)\right) \neq(e, s) \mid e \leftarrow \chi_{e}, s \leftarrow \chi_{s}\right]$

is the decoding failure rate for given $\left(\mathbf{B}_{f, g}, w\right)$. In fact, it is hard to numerically compute $\mu\left[P\left(\mathbf{B}_{f, g}, w, \chi_{s}, \chi_{e}\right)\right]$, since the distribution of $\left(G^{\prime}, F^{\prime}\right)$ (defined in Claim 4) is complicated. It is however easy to numerically compute $P\left(\mathbf{B}_{f, g}, w, \chi_{s}, \chi_{e}\right)$ given $\left(\mathbf{B}_{f, g}, w\right)$.

We experimentally calculate $P\left(\mathbf{B}_{f, g}, w, \chi_{s}, \chi_{e}\right)$ for some $\left(\mathbf{B}_{f, g}, w\right)$ generated with the suggested parameters (see Tables 3 and 4 ). The failure probabilities are smaller than for a naive Gaussian model, and significantly so for a ring dimension of 256.

\section{BAT KEM}

In this section, we present a KEM scheme, called BAT, constructed following the aforementioned encode/decode paradigm. Its secret key is an NTRU trapdoor basis as in the Falcon signature. As a consequence, some codes for Falcon implementation can be reused.

BAT permits very compact parameters. Specifically, the modulus $q$ is greatly reduced in contrast to Falcon. More remarkably, the ciphertext is well compressed: each coefficient requires only less than one byte of storage.

\subsection{Algorithm description}

Prior to the description of our KEM, we first present the underlying public key encryption. It is specified by the following parameters:

- $\mathcal{R}=\mathbb{Z}[x] /\left(x^{n}+1\right)$ with $n=2^{l}$.

- $q=b k+1$ with $b, k \in \mathbb{N}$. Note that $b$ determines the size of each ciphertext coefficient and $k$ determines the decoding distance.

- $q^{\prime} \in \mathbb{N}$ is used to control the decryption failure rate.

At a high level, our idea is to build an encryption scheme upon a one-way trapdoor function. Indeed, for a pseudorandom public key $h$, the function $F(s, e)=h s+e \bmod q$ is one-way under the Ring-LWE assumption, but one can invert it with the trapdoor $\mathbf{B}_{f, g}$ as shown in Section 3. In our scheme, the encryption is to compute $c=F(s, e)$ and the decryption is to recover $s$ by inverting $F(s, e)$.

The key generation is shown in Algorithm 4.1. The first step is to generate an NTRU trapdoor basis $\mathbf{B}_{f, g}$ along with the public key $h$. This is similar to the Falcon key generation, but the size of the secret key is changed. The second step pre-computes an auxiliary 
vector $w$. As explained in Section 3, w is used for decoding a larger error while avoiding floating-point arithmetic in the decapsulation. We include it as a part of the secret key. Note that Falcon key generation also pre-computes the Falcon tree for signing, but that computation is useless in our scheme.

The encryption algorithm is described in Algorithm 4.3. The message space is $\mathcal{M}=$ $\{0,1\}^{\lambda}$ where $\lambda$ denotes the claimed security level. The encryption is obtained by applying a simple worst-case to average-case correctness (ACWC for short) transform, introduced by a concurrent work [DHK $\left.{ }^{+} 21\right]$, on a deterministic encryption (Algorithm 4.2). Thanks to the ACWC transform, the encryption scheme achieves the IND-CPA security and its decryption failure rate is independent of the message. The base encryption (Algorithm 4.2) is to compute the trapdoor function on an ephemeral $s$. For better compactness, we replace $F(s, e)$ with $F(s)=\left[\frac{(h s \bmod q)}{k}\right]$ and thus use Ring-LWR as the hardness assumption. It is easy to see that the storage of a ciphertext is $n \log b+\lambda$ bits. The decryption stems from the decoding algorithm in Section 3. The formal description is provided in Algorithm 4.4.

Remark 1. The work [DHK $\left.{ }^{+} 21\right]$ also introduces an ACWC transform avoiding the $\lambda$-bit overhead on the ciphertext. That transform is not so direct and the analysis is more complicated.

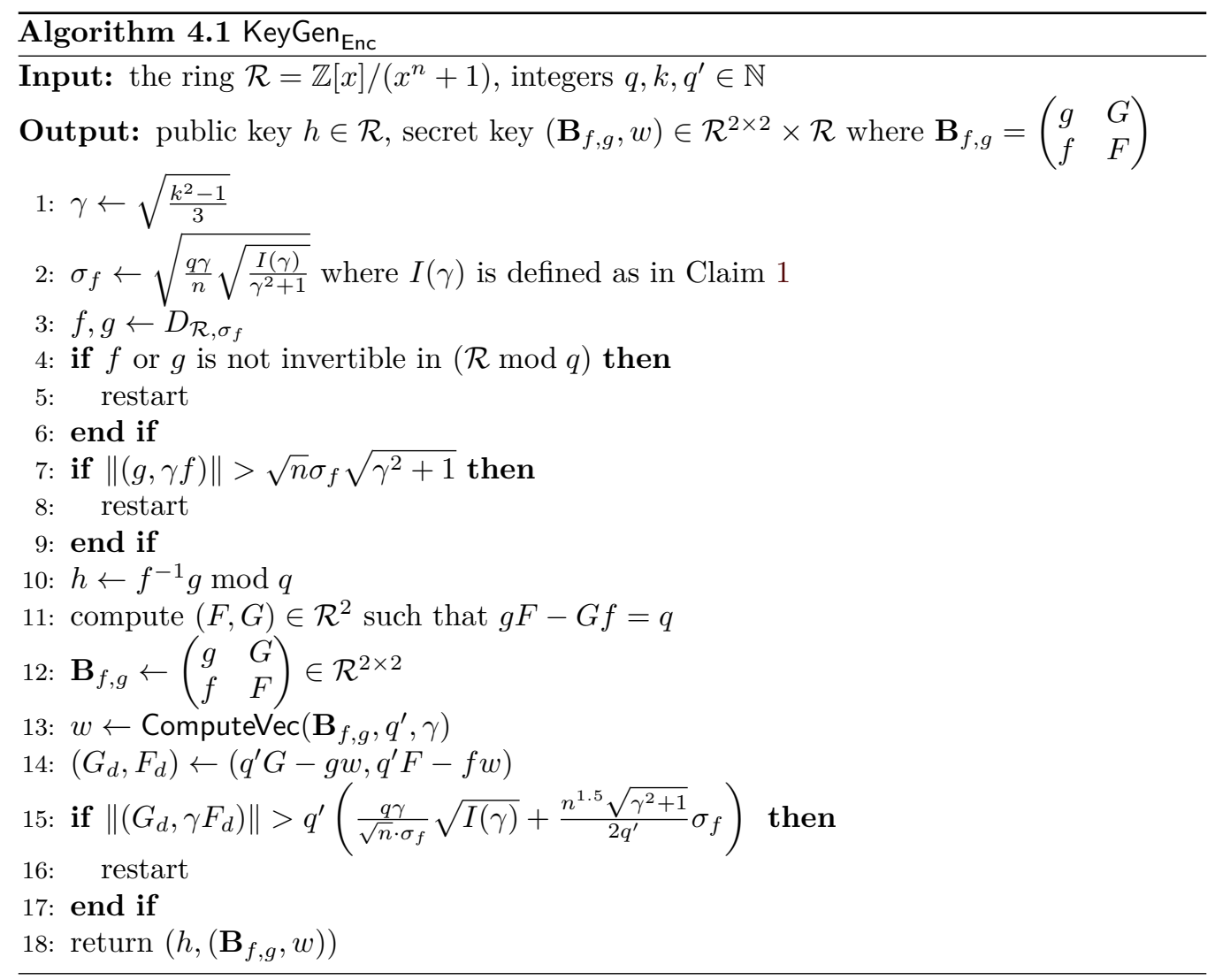



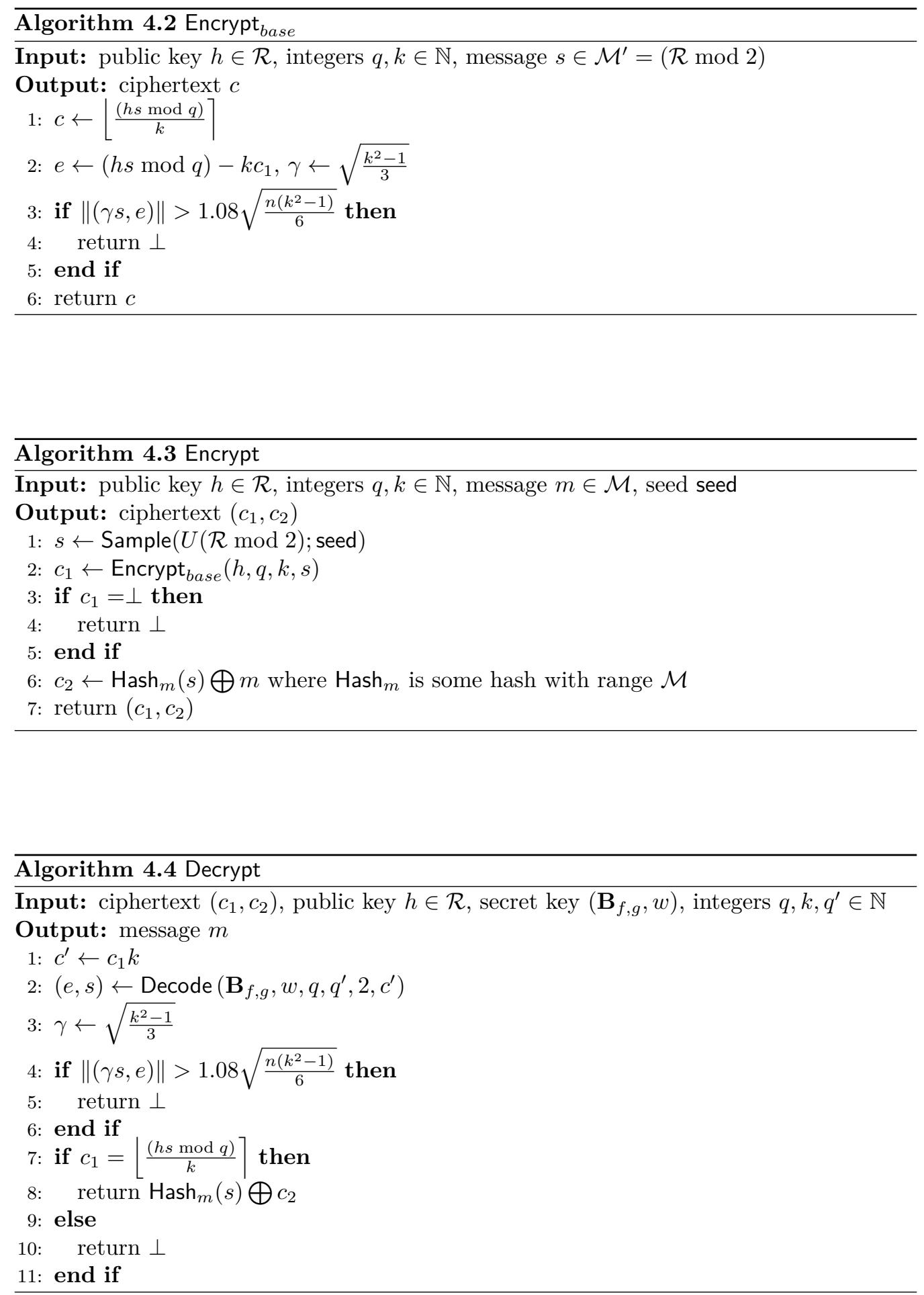

By some standard techniques [FO99, Den03], an IND-CCA secure KEM immediately follows from our IND-CPA encryption. Algorithms 4.5 and 4.6 describe the encapsulation and decapsulation algorithms respectively, in which $\mathcal{S}$ (resp. $\mathcal{K}$ ) is the set of seed (resp. shared key). The detailed security arguments are given in Section 5.2. 

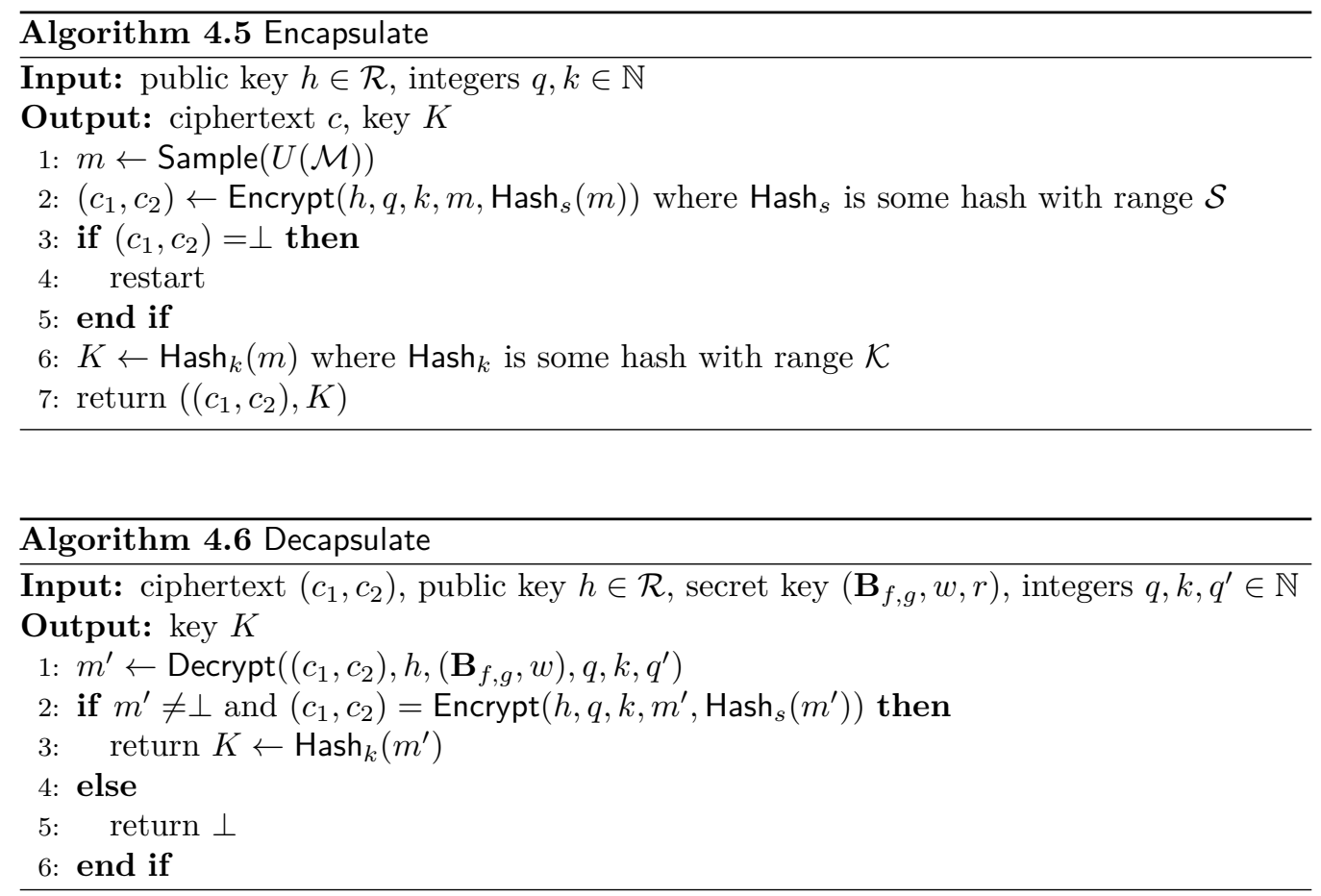

\subsection{Parameter selection}

We keep the same ring $\mathcal{R}$ as Falcon but choose a much smaller modulus $q$. Indeed, a smaller modulus forbids the applications of a complete NTT, nevertheless similar techniques [LS18, LS19] still allow a very fast polynomial multiplication. For security, a smaller $q$ implies a smaller standard deviation of the secret key distribution and then less entropy of the secret key. However such loss does not reduce the concrete security level much.

A notable modification exists in key generation. In Falcon, $(f, g)$ is sampled to make $\|(g, f)\| \approx\left\|\left(G^{*}, F^{*}\right)\right\|$ where $\left(G^{*}, F^{*}\right)$ is the Gram-Schmidt orthogonalization of $(G, F)$ in $\mathbf{B}_{f, g}$. However, in BAT, we choose $\sigma_{f}$ satisfying $\sqrt{n} \sigma_{f} \sqrt{\gamma^{2}+1}=\frac{q \gamma}{\sqrt{n} \cdot \sigma_{f}} \sqrt{I(\gamma)}$. This gives rise to a nearly optimal decryption failure rate according to Claim 1 . Particularly, when $\gamma=1$, the $\sigma_{f}$ we use also makes $\|(g, f)\| \approx\left\|\left(G^{*}, F^{*}\right)\right\|$ as the case of Falcon. Yet for this case, $\sigma$ used by BAT is different from that by Falcon, which is explained in Remark 5.

Let us recall that $c^{\prime}=c k=h s-k\left(\frac{h s}{k}-\left\lfloor\frac{(h s \bmod q)}{k}\right\rceil\right):=h s+e \bmod q$. We model $e$ drawn from $U\left(\mathbb{Z}_{k}^{n}\right)$ and thus ${ }^{3} \sigma_{e}=\sqrt{\frac{k^{2}-1}{12}}$. As $\sigma_{s}=\frac{1}{2}$, it follows that $\gamma=\sigma_{e} / \sigma_{s}=\sqrt{\frac{k^{2}-1}{3}}$. Our KEM tolerates a small decryption failure rate for better performance, as many current lattice-based KEMs. The decryption failure rate is equal to the probability of incorrect decoding. According to Claim 1, it is heuristically bounded by $2 n \cdot(1-\operatorname{erf}(\tau))$ for some $\tau$. We bound the size of the error used to 1.08 its average to limit the impact of precomputed messages on the decryption failure rates: the exponent may be reduced by $20 \%$ in the worst case. As a contrast and verification, we also numerically computed the decryption failure rate for 100 keys in practice: the standard deviation of the logarithm of the rate over the secret key distribution is around 8 , and the computed values are even smaller than their heuristic estimates. Therefore we present the numerical values (the average for randomly generated keys) for the decryption failure rate and the tail-bound parameter $\tau$ for the heuristic estimates.

\footnotetext{
${ }^{3}$ The actual distribution is within statistical distance $1 / q$ of $U\left(\mathbb{Z}_{k}^{n}\right)$.
} 
Table 3 shows the suggested parameters.

Table 3: Suggested parameters for BAT.

\begin{tabular}{|c|c|c|c|c|c|c|}
\hline & $n$ & $(b, k, q)$ & $\sigma_{f}$ & $q^{\prime}$ & $\tau$ & Decryption Failure \\
\hline BAT-512 & 512 & $(128,2,257)$ & 0.596 & 64513 & 9.46 & $2^{-146.7}$ \\
BAT-1024 & 1024 & $(192,4,769)$ & 0.659 & 64513 & 10.42 & $2^{-166.7}$ \\
\hline
\end{tabular}

The parameter set for lightweight BAT. We further suggest one more parameter set particularly aiming at a lower security level (around 80 bits of security), which may be of interest for some lightweight use-cases. We call this lightweight variant LW-BAT. In LW-BAT, the degree $n$ is only 256 ; for better compactness the modulus $q$ does not support NTT anymore. We choose a relatively high decryption failure rate $2^{-71.9}$, but it should be sufficient for lightweight applications, e.g. IoT: for the Round5 IoT parameters $\left[\mathrm{BBC}^{+} 20\right]$, the decryption failure rate is $2^{-41}$ even larger than ours. Table 4 summarizes the concrete parameter set.

Table 4: Suggested parameters for LW-BAT.

\begin{tabular}{|c|c|c|c|c|c|c|}
\hline & $n$ & $(b, k, q)$ & $\sigma_{f}$ & $q^{\prime}$ & $\tau$ & Decryption Failure \\
\hline LW-BAT & 256 & $(64,2,128)$ & 0.595 & 64513 & 6.71 & $2^{-71.9}$ \\
\hline
\end{tabular}

\section{Security}

We now report on the security of BAT. First, we demonstrate the IND-CCA security of our KEM under some hardness assumptions. Then we estimate the concrete security according to the best known attacks.

\subsection{Assumptions}

The (decision) NTRU assumption. Let $\mathcal{R}_{q}^{\times}$be the set of invertible elements in $\mathcal{R} / q \mathcal{R}$. Let $\chi$ be some distribution over $\mathcal{R}_{q}^{\times}$. The advantage of adversary A in solving the decision NTRU problem $\mathrm{NTRU}_{\mathcal{R}, q, \chi}$ is

$\operatorname{Adv}_{\mathcal{R}, q, \chi}^{\mathrm{NTRU}}(\mathrm{A})=\left|\operatorname{Pr}\left[b=1 \mid f, g \hookleftarrow \chi ; b \leftarrow \mathrm{A}\left(f^{-1} g \bmod q\right)\right]-\operatorname{Pr}\left[b=1 \mid u \hookleftarrow U\left(\mathcal{R}_{q}^{\times}\right) ; b \leftarrow \mathrm{A}(u)\right]\right|$.

In our case, $\mathcal{R}=\mathbb{Z}[x] /\left(x^{n}+1\right)$ and $\chi$ is the distribution of the secret key $f$ and $g$.

Remark 2. There are some researches on the hardness of the decision NTRU assumption over $\mathcal{R}=\mathbb{Z}[x] /\left(x^{n}+1\right)$. Notably, as shown in [SS11], when $\chi$ is a discrete Gaussian of standard deviation $\sigma=\widetilde{\omega}(n \sqrt{q})$, the ratio of $f$ and $g$ is statistically indistinguishable from uniform, which gives a firm theoretical grounding. The decision NTRU assumption with a narrow distribution $\chi$ is also closely related to Falcon $\left[\mathrm{FHK}^{+} 20\right]$ and sometimes referred to as the Decisional Small Polynomial Ratio (DSPR) assumption [LATV12, BD20].

The (search) Ring-LWR assumption. Let $\chi$ be some distribution over $\mathcal{R}$. The advantage of adversary $A$ in solving the search Ring-LWR problem $\operatorname{RLWR}_{\mathcal{R}, q, k, \chi}$ is

$$
\operatorname{Adv}_{\mathcal{R}, q, k, \chi}^{\mathrm{RLWR}}(\mathrm{A})=\underset{a \hookleftarrow U\left(\mathcal{R}_{q}^{\times}\right), s \hookleftarrow \chi}{\operatorname{Pr}}\left[\mathrm{A}\left(a,\left\lfloor\frac{(\text { as } \bmod q)}{k}\right\rceil\right)=s\right] .
$$

In our case, $\mathcal{R}=\mathbb{Z}[x] /\left(x^{n}+1\right)$ and $\chi=U(\mathcal{R} \bmod 2)$. 
Remark 3. The theoretical foundation of the search Ring-LWR assumption is developed in [BPR12, $\mathrm{BGM}^{+} 16, \mathrm{CZZ18}$. There are also some practical schemes, e.g. Lizard [CKLS18] and Saber $\left[\mathrm{BMD}^{+} 20\right]$, using the Ring-LWR over $\mathbb{Z}[x] /\left(x^{n}+1\right)$ or its module variant as their hardness assumption. Indeed, the provable hardness of Ring-LWR with a binary secret $s$ remains open. Yet this would not weaken the concrete security as per the state-of-the-art cryptanalysis results, especially when $q$ is relatively small (as in our case).

\subsection{KEM security}

The security notion we prove for BAT is IND-CCA security (indistinguishability against chosen-ciphertext attacks). To this end, we first note that the underlying encryption (Algorithms 4.3 and 4.4) is IND-CPA secure (indistinguishability against chosen-plaintext attacks) under the assumptions in Section 5.1.

Theorem 2. Let $\Pi$ be the public key encryption scheme defined by Algorithms 4.1, 4.3 and 4.4. For any adversary $\mathrm{A}$, there exist adversaries $\mathrm{A}_{1}$ and $\mathrm{A}_{2}$ of roughly the same running time as that of $\mathrm{A}$ such that

- $\operatorname{Adv}_{\Pi}^{I N D-C P A}(\mathrm{~A}) \leq A d v$, when Hash ${ }_{m}$ is a classical random oracle;

- $\operatorname{Adv}_{\Pi}^{I N D-C P A}(\mathrm{~A}) \leq 2 d_{H} \sqrt{\operatorname{Adv}}$, when $\mathrm{A}$ is a quantum adversary, Hash ${ }_{m}$ is a quantumaccessible random oracle and $d_{H}$ is the query depth.

where $A d v=A d v_{\mathcal{R}, q, \chi_{1}}^{N T R U}\left(\mathrm{~A}_{1}\right)+\operatorname{Ad} v_{\mathcal{R}, q, k, \chi_{2}}^{R L W R}\left(\mathrm{~A}_{2}\right), \chi_{1}$ is the distribution of the secret key $(f, g)$ and $\chi_{2}=U(\mathcal{R} \bmod 2)$.

Proof. Let $\Pi_{\text {base }}$ be the public key encryption scheme defined by Algorithms 4.1 and 4.2. We first show the OW-CPA security of $\Pi_{\text {base }}$. Let $\operatorname{Pr}\left[W_{i}\right]$ be the probability of the adversary winning Game $i$. Game 0 is the classical OW-CPA game, thus $\operatorname{Pr}\left[W_{0}\right]=\operatorname{Adv}_{\Pi_{b a s e}}^{\mathrm{OW}-\mathrm{CPA}}(\mathrm{A})$. Game 1 and Game 0 only differ in the key generation: Game 1 samples the public key $h$ from $U\left(\mathcal{R}_{q}^{\times}\right)$instead. We exploit the different distributions of $h$ in Game 0 and Game 1 to construct $\mathrm{A}_{1}$ for the decision NTRU problem. Upon input $h \in \mathcal{R}_{q}^{\times}, \mathrm{A}_{1}$ plays the challenger in Game 0 except for setting $p k=h$ directly, and claims that $h$ is an NTRU public key if $\mathrm{A}$ wins. Therefore $\operatorname{Adv}_{\mathcal{R}, q, \chi_{1}}^{\mathrm{NTRU}}\left(\mathrm{A}_{1}\right)=\left|\operatorname{Pr}\left[W_{0}\right]-\operatorname{Pr}\left[W_{1}\right]\right|$. We then define $\mathrm{A}_{2}$ for the search Ring-LWR problem. Upon input $(h, c), \mathrm{A}_{2}$ plays the challenger in Game 1 except for setting $p k=h$ and $c t=c$. It is easy to see that $\operatorname{Adv}_{\mathcal{R}, q, k, \chi_{2}}^{\mathrm{RLWR}}\left(\mathrm{A}_{2}\right)=\operatorname{Pr}\left[W_{1}\right]$. It immediately follows that $\operatorname{Adv}_{\Pi_{b a s e}}^{\text {OW-CPA }}(\mathrm{A}) \leq \mathrm{Adv}$.

The scheme $\Pi$ is derived from $\Pi_{b a s e}$ by utilizing a simple ACWC transform $\left[\mathrm{DHK}^{+} 21\right]$. Note that in the NTRU setting, preimage resistance (called PRE-CPA security) is essentially equivalent to OW-CPA security. Combining $\left[\mathrm{DHK}^{+} 21\right.$, Lemma 2.2] and $\left[\mathrm{DHK}^{+} 21\right.$, Theorem 3.3] (resp. [DHK ${ }^{+}$21, Theorem 3.4]), the claim in the ROM (resp. QROM) follows.

The BAT KEM is obtained via applying a Fujisaki-Okamoto transform [Den03] to the IND-CPA secure encryption. Theorem 3 gives the concrete security statement of the IND-CCA security of BAT. Theorem 3 is adapted from [DHK ${ }^{+} 21$, Theorems 2.4 and 2.5] that was first developed in [HHK17] and [DFMS21].

Theorem 3. Let $\Pi$ be the public key encryption scheme defined by Algorithms 4.1, 4.3 and 4.4. Let $\Pi_{C C A}$ be the IND-CCA secure KEM defined by Algorithms 4.5 and 4.6. Let $\mathcal{M}$ be the message space, $\delta$ be the decryption error rate and $\gamma$ be the weakly spread parameter of $\Pi .^{4}$ For any adversary A making at most $q_{D}$ decapsulation, $q_{H}$ hash (i.e. Hash ${ }_{k}$ and Hash $_{s}$ ) queries, against the IND-CCA security of $\Pi_{C C A}$, there exists an adversary $\mathrm{A}^{\prime}$ against the IND-CPA security of $\Pi$ such that

\footnotetext{
${ }^{4}$ The definitions of $\delta$ and $\gamma$ are specified in [HHK17] and [DFMS21].
} 
- $A d v_{\Pi_{C C A}}^{I N D-C C A}(\mathrm{~A}) \leq 2\left(A d v_{\Pi}^{I N D-C P A}\left(\mathrm{~A}^{\prime}\right)+q_{H} /|\mathcal{M}|\right)+q_{D} \cdot 2^{-\gamma}+q_{H} \delta$, $\boldsymbol{T i m e}\left(\mathrm{A}^{\prime}\right) \approx$ Time(A), when Hash $_{k}$ and Hash are classical random oracles;

- $A d v_{\Pi_{C C A}}^{I N D-C C A}(\mathrm{~A}) \leq 2 q_{T} \sqrt{A d v_{\Pi}^{I N D-C P A}\left(\mathrm{~A}^{\prime}\right)}+24 q_{T}^{2} \sqrt{\delta}+24 \sqrt{q_{T}^{3} q_{D}} \cdot 2^{-\gamma / 4}$ with $q_{T}=$ $2\left(q_{D}+q_{H}\right)$, Time $\left(\mathrm{A}^{\prime}\right) \approx$ Time $(\mathrm{A})+O\left(q_{H} \cdot q_{D} \cdot\right.$ Time $($ Encrypt $\left.)+q_{T}^{2}\right)$, when $\mathrm{A}$ is a quantum adversary, $\mathrm{Hash}_{k}$ and $\mathrm{Hash}_{s}$ are quantum-accessible random oracles.

\subsection{Concrete security}

We estimate the concrete security using the usual cryptanalytic methodology of assessing the cost of the best attacks against key recovery and message recovery. For BAT, the cost of key recovery relies on the hardness of NTRU, while the cost of message recovery relies on the hardness of Ring-LWR. Thus we respectively analyze the costs of key recovery and message recovery based on the primal attack [ADPS16] that is a fundamental cryptanalysis method in lattice-based cryptography $\left[\mathrm{ACD}^{+} 18\right]$. For BAT, the primal attack indeed leads to better security estimates than other attacks.

\subsubsection{Cost of lattice reduction}

We begin with a brief introduction to lattice reduction that is heavily used by the primal attack. Currently, the most practical lattice reduction algorithms are BKZ [SE94] and BKZ 2.0 [CN11]. Let BKZ- $\beta$ denote the BKZ/BKZ 2.0 with blocksize $\beta$. The cost of running BKZ- $\beta$ on a $d$-dimensional lattice is estimated by $C_{\mathrm{BKZ}-\beta}=t \cdot d \cdot C_{\mathrm{SVP}-\beta}$ where $t$ is the tour number BKZ- $\beta$ takes and $C_{\mathrm{SVP}-\beta}$ is the cost of solving SVP on a $\beta$-dimensional lattice. We follow a typical setting:

$$
t=1, \quad C_{\mathrm{SVP}-\beta}= \begin{cases}2^{0.292 \beta} & \text { Classical SVP solver [BDGL16] } \\ 2^{0.257 \beta} & \text { Quantum SVP solver [CL21] }\end{cases}
$$

Remark 4. The BKZ cost model we use is not extremely conservative: some lattice-based schemes use the Core-SVP model in which $C_{\mathrm{BKZ}-\beta}=2^{0.292 \beta}$ (resp. $2^{0.257 \beta}$ ) for classical (resp. quantum) setting. Nevertheless the used SVP cost models are quite conservative and provide a safe margin: they ignore the lower order term $o(\beta)$ in the exponent, which is substantial for concrete security. For a fair comparison, we shall also show the required blocksize $\beta$ along with the estimated cost.

\subsubsection{Primal attack}

The primal attack consists of constructing a uSVP (unique-SVP) instance and solving it by lattice reduction. We refer to [ADPS16, AGVW17, $\left.\mathrm{ACD}^{+} 18\right]$ for details.

For key recovery, the uSVP instance is $\left(\begin{array}{cc}q \mathbf{I}_{n} & M_{n}(h) \\ \mathbf{I}_{n}\end{array}\right) \in \mathbb{Z}^{2 n \times 2 n}$ where $M_{n}(h)$ is the matrix form of the public key $h$. The secret key pair $\left(\begin{array}{l}g \\ f\end{array}\right)$ is a short vector of the uSVP instance. To optimize the primal attack, one can reduce the instance dimension by "forgetting" some equations and take homogeneousness into account.

For message recovery, it suffices to recover $s$ from $c_{1}=\left\lfloor\frac{(h s \bmod q)}{k}\right\rceil$. Let $c=k \cdot c_{1}$, then $c=h s-k\left(\frac{h s}{k}-\left\lfloor\frac{(h s \bmod q)}{k}\right\rceil\right):=h s+e \bmod q$. We construct the uSVP instance as $\left(\begin{array}{ccc}q \mathbf{I}_{n} & -M_{n}(h) & \operatorname{coef}(c) \\ & \mathbf{I}_{n} & 1\end{array}\right) \in \mathbb{Z}^{(2 n+1) \times(2 n+1)}$ that contains $\left(\begin{array}{l}e \\ s \\ 1\end{array}\right)$ as a short vector. Unlike the case of key recovery, the unknowns $s$ and $e$ have different distributions. Therefore, the 
primal attack can be improved by re-scaling technique. Also, the strategy of "forgetting" some equations still works here.

The primal attack with all above optimizations is systematically discussed in [DSDGR20]. We estimate the cost of primal attack with the open-source script ${ }^{5}$ of [DSDGR20]. Numbers are shown in Table 5 .

Table 5: Concrete security estimate for BAT.

\begin{tabular}{|c|c|c|c|c|}
\hline & LW-BAT & BAT-512 & BAT-1024 \\
\hline \multicolumn{2}{|c|}{ Target security level } & lightweight $^{\mathrm{a}}$ & NIST-I & NIST-V \\
\hline \multirow{3}{*}{ Key recovery } & ( BKZ blocksize & 236 & 475 & 933 \\
& Classical security & 77.8 & 148.5 & 283.2 \\
Quantum security & 69.5 & 131.9 & 250.6 \\
\hline \multirow{2}{*}{ Message recovery } & ( BKZ blocksize & 219 & 447 & 949 \\
& Classical security & 72.8 & 140.3 & 287.8 \\
& Quantum security & 65.2 & 124.7 & 254.6 \\
\hline
\end{tabular}

${ }^{a}$ Around 80 bits of classical security. Due to the conservative SVP hardness and the heavy memory cost of sieving, LW-BAT arguably reaches this level.

\subsubsection{Other attacks}

We list some other known attacks, while they do not outperform the primal attack for the proposed parameter sets.

Hybrid attack. This attack is a combination of lattice reduction and meet-in-themiddle techniques. It was proposed as an improved attack against early NTRU [HG07] and further studied in [BGPW16, Ngu21, SC19]. The hybrid attack is effective for the NTRU or LWE problem with particularly sparse secret or error vectors. The secret and error in BAT have enough entropy to resist this attack.

Dual attack. This attack is proposed to solve decision LWE problem and thus does not apply to BAT that is actually an NTRU-type scheme [ACD $\left.{ }^{+} 18\right]$.

Learning attacks. This kind of attacks [NR06, DN12] were proposed to break insecure NTRU signatures $\left[\mathrm{HHP}^{+} 03\right]$ in which signature transcripts leak secret information of the NTRU trapdoor. While BAT uses NTRU trapdoor as the secret key, the ciphertext is indistinguishable from uniform under the Ring-LWR assumption. Therefore, BAT resists to learning attacks.

Overstretched NTRU attacks. These attacks [ABD16, KF17] only work when the modulo $q$ is significantly larger than the NTRU secret coefficients. This is not the case of BAT.

Algebraic attacks. There is a rich algebraic structure in BAT. While there are some results exploiting this algebraic structure [KEF20] to speed up lattice reduction, the gains with respect to their general lattice equivalent are no more than polynomial factors.

\section{Implementation Details}

We implemented BAT with integer-only computations. We provide here some details on the used techniques. Our implementation is available at: https://github.com/pornin/BAT/

\footnotetext{
${ }^{5}$ https://github.com/lducas/leaky-LWE-Estimator
} 


\subsection{Key pair generation}

Key pair generation starts with producing the short polynomials $f$ and $g$, then solving the NTRU equation to obtain $F$ and $G$. This specific step uses the algorithm described in [PP19]. Compared with the reference implementation of Falcon, the following differences are noteworthy:

- BAT polynomials have a lower norm than their Falcon equivalent. The polynomial resultants obtained at the deepest level of the recursive algorithm are then shorter, which improves performance.

- All uses of floating-point operations (for Babai's nearest-plane algorithm) have been replaced with fixed-point values (over 64 bits, with 32 fractional bits), which removes all dependencies on the floating-point unit. Since fixed-point values have a limited range, this implies that the reduction may fail, leading to a key pair generation restart. Failed cases can be efficiently filtered out early in the process by checking the current partial solution to the NTRU equation modulo a small prime integer; hence, the overhead implied by these restarts is low. At degree $n=512$, about $30 \%$ of candidate $(f, g)$ pairs lead to a restart.

- Some memory reorganization allowed for additional RAM savings, down to 12288 and 24576 bytes for $n=512$ and 1024, respectively (compared to 14336 and 28672 bytes for Falcon).

Once the complete NTRU basis $(f, g, F, G)$ has been obtained, ComputeVec is used to obtain $w$. The polynomials $\gamma^{2} F \bar{f}+G \bar{g}$ and $\gamma^{2} f \bar{f}+g \bar{g}$ are first computed modulo a small prime where NTT can be applied for efficient computations (a 31-bit prime is used; since the basis coefficients are all small, it is easily seen that coefficients do not exceed $2^{19}$ in absolute value). The $v$ polynomial is then obtained by performing the division in the FFT domain, using the same fixed-point code as the one used for solving the NTRU equation. The division itself is performed with a constant-time bit-by-bit routine.

Since fixed-point values are approximations of the real coefficients of $v$, the rounding step may occasionally be wrong by 1 . Extensive tests show that it is a relatively uncommon occurrence (it happens in about $0.5 \%$ of keys at $n=512$ ) and always when $v$ is close to $z+1 / 2$ for some integer $z$; over 30000 random key pairs, the largest observed deviation of $v-1 / 2$ from the closest integer, for coefficients where our implementation rounds to $w$ incorrectly, is lower than $2 \times 10^{-4}$. This means that in all observed cases, $\left|w_{i}-v_{i}\right|<$ $1 / 2+2 \times 10^{-4}$. Since the decoding process works as long as $\left|w_{i}-v_{i}\right|<1$, the impact on the decryption failure rate is negligible.

\subsection{Field operations}

Efficient and secure (constant-time) operations in the small base fields (modulo $q$ and $q^{\prime}$ ) are implemented with Montgomery multiplication. Namely, a value $x$ modulo $q$ is represented by an integer $y$ in the 1 to $q$ range (inclusive), such that $y=2^{32} x \bmod q$. Montgomery reduction can be implemented in two 16-bit multiplications, two shifts and one addition; they can moreover be mutualized because analysis shows that reduction works properly for values up to close to $2^{32}$. For details on this technique, see [Por20].

On recent x 86 platforms, SIMD opcodes can be used to further optimize operations. AVX2 registers can store 16 values modulo $q$ (or $q^{\prime}$ ) and perform 16 Montgomery reductions in parallel. The _mm256_mullo_epi16() and_mm256_mulhi_epu16() intrinsics compute, respectively, the low and high halves of a 16-bit product, with a very low reciprocal throughput (0.5 cycles). Computing 16 modular multiplications in parallel requires in total only 6 invocations of such intrinsics. 


\subsection{NTT multiplication}

Since $q-1$ and $q^{\prime}-1$ are multiples of 256 for BAT, the NTT can be applied to speed up computations over polynomials modulo $X^{n}+1$, when working with integers modulo either $q$ or $q^{\prime}$. For $n$ a power of two up to $2^{7}=128$, the NTT representation of a polynomial $f$ is the set of $f\left(\zeta^{2 i+1}\right)$ for $0 \leq i \leq n-1$, where $\zeta$ is a primitive $2 n$-th root of 1 modulo $q$ ( or $q^{\prime}$ ). In NTT representation, addition and multiplication of polynomials can be done coefficient-wise, hence with cost $O(n)$ operations modulo $q$. Moreover, conversion to and from NTT representation can be done in $O(n \log n)$ steps.

For larger degrees, we cannot use full NTT representation, but we can still optimize operations by splitting polynomials as follows. Consider $n=512$; the polynomial $f$ modulo $X^{512}+1$ can be split into four sub-polynomials as follows:

$$
f=f_{0}\left(X^{4}\right)+X f_{1}\left(X^{4}\right)+X^{2} f_{2}\left(X^{4}\right)+X^{3} f_{3}\left(X^{4}\right)
$$

the polynomials $f_{i}$ being of degree up to 127 , and operating modulo $X^{128}+1$. Then, operations on such polynomials can be expressed as a relatively small number of operations on the sub-polynomials, which themselves can be implemented in the NTT domain, since the sub-polynomials are of degree less than 128.

In our implementation, the NTT representations of the sub-polynomials are interleaved, so as to maximize parallelization efficiency.

\subsection{Polynomial splitting and Karatsuba multiplication}

For LW-BAT, we use $q=128$, which prevents us from using the NTT straightforwardly ${ }^{6}$. Instead, for polynomial multiplications, we use Karatsuba with an even/odd split, by writing a polynomial $f$ as: $f=f_{0}\left(X^{2}\right)+X f_{1}\left(X^{2}\right)$ with $f_{0}$ and $f_{1}$ being half-size polynomials (they operate modulo $X^{n / 2}+1$ ). We can then express the product of $f$ and $g$ as:

$$
f g=\left(f_{0} g_{0}\right)\left(X^{2}\right)+X^{2}\left(f_{1} g_{1}\right)\left(X^{2}\right)+X\left(\left(f_{0}+f_{1}\right)\left(g_{0}+g_{1}\right)-f_{0} g_{0}-f_{1} g_{1}\right)\left(X^{2}\right)
$$

i.e. we turn the multiplication of two polynomials modulo $X^{n}+1$ into three multiplications of polynomials modulo $X^{n / 2}+1$. We use this reduction recursively, until polynomials have degree less than 4.

The same split is used to compute polynomial divisions modulo $X^{n}+1$ : this is used to compute the public key $h=g / f \bmod q$, and also to rebuild $G$ from $f, g$ and $F$ when the short format for private key storage was used. The even-odd split allows us to write:

$$
\frac{1}{f}=\frac{f_{0}\left(X^{2}\right)-X f_{1}\left(X^{2}\right)}{\left(f_{0}\left(X^{2}\right)+X f_{1}\left(X^{2}\right)\right)\left(f_{0}\left(X^{2}\right)-X f_{1}\left(X^{2}\right)\right)}=\frac{f_{0}\left(X^{2}\right)-X f_{1}\left(X^{2}\right)}{\left(f_{0}^{2}+X f_{1}^{2}\right)\left(X^{2}\right)}
$$

which reduces inversion modulo $X^{n}+1$ to a multiplication (modulo $X^{n}+1$ ) and an inversion modulo $X^{n / 2}+1$. Applied recursively, this method leads us to the simple problem of inverting an integer modulo $q=128$, which can be done in a few inexpensive multiplications.

\subsection{Decoding}

Decoding involves computing polynomials with integer coefficients modulo $q, q^{\prime}$ and $Q$. The final step requires solving for $e^{\prime}$ and $s^{\prime}$; we only need $s^{\prime}$ in practice, since we can use encapsulation to verify the result. Moreover, there are only two possible values for each coefficient of $s^{\prime}$ (for $1 / 2$ and $-1 / 2$ ) and we merely need to disambiguate between these two

\footnotetext{
${ }^{6}$ A very recent work $\left[\mathrm{CHK}^{+} 21\right]$ shows that by introducing an extra prime $p$ such that $p>n q^{2} / 2$ and $p=1 \bmod 2 n$, one can implement the multiplication over $\mathcal{R}_{q}$ with NTT over $\mathcal{R}_{p}$.
} 
values. To keep to integer values, we do not recover $s^{\prime}$ but $q q^{\prime} Q s^{\prime}$; moreover, we perform computations modulo an additional small prime distinct from $q$ and $q^{\prime}$. In practice, when $q=257$, we perform the last step by working modulo 769 ; when $q=128$ or 769 , we use computations modulo 257.

\subsection{Encoding and storage}

We defined compact encoding formats for public keys, private keys, and ciphertexts. Each format starts with a single header byte which identifies the object type and parameter set.

Public keys are polynomials with coefficients modulo $q$. When $q=257$, we encode coefficients by groups of eight, each group using 65 bits: each coefficient is split into a low half ( 4 bits) and a high half (value 0 to 16, inclusive); eight "high halves" are encoded over 33 bits in base 17. For $q=769$, a similar mechanism is used, with 5 coefficients being encoded over 48 bits. All encoding and decoding operations can be implemented with only simple 32-bit multiplications, and can be done efficiently in a constant-time manner (this last property does not nominally matter for public keys, which are public).

Ciphertexts are mainly polynomials with small, signed integer coefficients. When $q=257$, coefficients of $c_{1}$ are in the -64 to +64 range; eight coefficients are encoded over 57 bits, in a way similar to public key encoding. For $q=769$, coefficients of $c$ are in the -96 to +96 range, and five coefficients are encoded over 38 bits in base 193 . The value $c_{2}$, which is a fixed-size binary value, is simply appended to the encoding of $c_{1}$.

Private keys have a "short" and a "long" formats. The long format includes the 32-byte seed that was used to generate $f, g$, and the 32-byte value $r$ (which is used when decapsulation fails). This seed is followed by a copy of $r$, then the polynomials $f, g, F, G$ and $w$ themselves, and the public key $h$. Coefficients of $f$ and $g$ are encoded over 4 bits each, in two's complement notation; for $F$ and $G, 6$ bits are used per coefficient, and 17 bits for $w$. The public key $h$ uses the same encoding as in the public key. The short format only stores the 32 -byte seed, and the polynomial $F$ : the value $r$ and the polynomials $f$ and $g$ are regenerated with the same pseudorandom (deterministic) process that was used during key pair generation; $G$ is recomputed using the NTRU equation (modulo $q$ ); and $w$ and $h$ are recomputed. While the short format is substantially shorter, decoding a private key stored in the short format has a nonnegligible overhead, but is still much cheaper than key pair generation, since the most expensive part (solving the NTRU equation from $f$ and $g$ alone) is avoided.

The numbers for required storages are listed in Table 6 .

Table 6: Storage requirements of BAT (in bytes, including the header byte).

\begin{tabular}{|c|c|c|c|c|}
\hline Variant & Public Key & $\begin{array}{c}\text { Ciphertext } \\
\text { (with FO) }\end{array}$ & $\begin{array}{c}\text { Private key } \\
\text { (short format) }\end{array}$ & $\begin{array}{c}\text { Private Key } \\
\text { (long format) }\end{array}$ \\
\hline LW-BAT & 225 & 203 & 225 & 1473 \\
BAT-512 & 521 & 473 & 417 & 2953 \\
BAT-1024 & 1230 & 1006 & 801 & 6030 \\
\hline
\end{tabular}

\subsection{Speed benchmarks}

We provide two implementations - (1) plain portable $\mathrm{C}$ version and (2) AVX2 version. We measured the speed on an Intel i5-8259U CPU clocked at $2.3 \mathrm{GHz}$; TurboBoost is disabled. Compiler is Clang-10.0, with optimization flags "-03". The AVX2 implementation uses intrinsic functions, and an additional optimization flag "-march=native". For key pair 
generation, the reported value is an average over several hundreds of key pairs ${ }^{7}$. For encapsulation, the reported value includes decoding of the public key, core encapsulation process with the FO transform, and ciphertext encoding to bytes; cost of generation of a random seed (of 10, 16 or 32 bytes, for the three BAT variants) from the operating system RNG is not included. For decapsulation, the value includes decoding of the ciphertext from bytes, and core decapsulation process with the FO transform. All hashing operations in the FO transform and for the PRNG used in key pair generation rely on the BLAKE2 function as specified in RFC 7693 (BLAKE2s for LW-BAT and BAT-512, BLAKE2b for BAT-1024) [SA15].

The timing data for two implementations are illustrated in Tables 7 and 8 respectively.

Table 7: Performance of the plain C implementation of BAT (in clock cycles).

\begin{tabular}{|c|c|c|c|}
\hline Variant & Key Generation & Encapsulation & Decapsulation \\
\hline LW-BAT & $\approx 23.3 \times 10^{6}$ & 81913 & 392062 \\
BAT-512 & $\approx 35.3 \times 10^{6}$ & 45473 & 290341 \\
BAT-1024 & $\approx 160.2 \times 10^{6}$ & 84666 & 552708 \\
\hline
\end{tabular}

Table 8: Performance of the AVX2 implementation of BAT (in clock cycles).

\begin{tabular}{|c|c|c|c|}
\hline Variant & Key Generation & Encapsulation & Decapsulation \\
\hline LW-BAT & $\approx 19.0 \times 10^{6}$ & 58263 & 240754 \\
BAT-512 & $\approx 29.4 \times 10^{6}$ & 11135 & 59745 \\
BAT-1024 & $\approx 145.6 \times 10^{6}$ & 25620 & 131162 \\
\hline
\end{tabular}

Acknowledgements. This work is supported by the National Natural Science Foundation of China (No. 62102216), the National Key Research and Development Program of China (Grant No. 2018YFA0704701), the Major Program of Guangdong Basic and Applied Research (Grant No. 2019B030302008) and Major Scientific and Techological Innovation Project of Shandong Province, China (Grant No. 2019JZZY010133).

\section{References}

[ABD16] Martin Albrecht, Shi Bai, and Léo Ducas. A subfield lattice attack on overstretched NTRU assumptions. In CRYPTO 2016, pages 153-178, 2016.

[ACD ${ }^{+}$18] Martin R Albrecht, Benjamin R Curtis, Amit Deo, Alex Davidson, Rachel Player, Eamonn W Postlethwaite, Fernando Virdia, and Thomas Wunderer. Estimate all the \{LWE, NTRU\} schemes! In SCN 2018, pages 351-367, 2018.

[AGVW17] Martin R. Albrecht, Florian Göpfert, Fernando Virdia, and Thomas Wunderer. Revisiting the Expected Cost of Solving uSVP and Applications to LWE. In ASIACRYPT 2017, pages 297-322, 2017.

\footnotetext{
${ }^{7}$ By nature, that process takes a varying time, since each candidate $(f, g)$ may or may not lead to a successful key pair generation; it is still "constant-time" in that timing variations are independent of the value of the private key which is ultimately generated.
} 
[ADPS16] Erdem Alkim, Léo Ducas, Thomas Pöppelmann, and Peter Schwabe. Postquantum Key Exchange-A New Hope. In USENIX Security 16, pages 327-343, 2016.

$\left[\mathrm{ABD}^{+} 20\right]$ Roberto Avanzi, Joppe Bos, Léo Ducas, Eike Kiltz, Tancrède Lepoint, Vadim Lyubashevsky, John M. Schanck, Peter Schwabe, Gregor Seiler, and Damien Stehlé. CRYSTALS-Kyber. 2020. https://pq-crystals.org/kyber/data/ kyber-specification-round3.pdf.

$\left[\mathrm{BBC}^{+} 20\right]$ Hayo Baan, Sauvik Bhattacharya, Jung Hee Cheon, Scott Fluhrer, Oscar Garcia-Morchon, Thijs Laarhoven, Rachel Player, Ronald Rietman, MarkkuJuhani Olavi Saarinen, Yongha Son, Ludo Tolhuizen, Jose Luis Torre Arce, and Zhenfei Zhang. Round5: KEM and PKE based on (Ring) Learning with Rounding. 2020. https://round5.org/doc/Round5_Submission042020. pdf.

[Bab86] László Babai. On Lovász' lattice reduction and the nearest lattice point problem. Combinatorica, 6(1):1-13, 1986.

[BPR12] Abhishek Banerjee, Chris Peikert, and Alon Rosen. Pseudorandom functions and lattices. In EUROCRYPT 2012, pages 719-737, 2012.

$\left[\mathrm{BMD}^{+} 20\right]$ Andrea Basso, Jose Maria Bermudo Mera, Jan-Pieter D'Anvers, Angshuman Karmakar, Sujoy Sinha Roy, Michiel Van Beirendonck, and Frederik Vercauteren. SABER: Mod-LWR based KEM. 2020. https://www.esat. kuleuven.be/cosic/pqcrypto/saber/files/saberspecround3.pdf.

[BDGL16] Anja Becker, Léo Ducas, Nicolas Gama, and Thijs Laarhoven. New directions in nearest neighbor searching with applications to lattice sieving. In SODA 2016, pages 10-24, 2016.

[BCLvV17] Daniel J. Bernstein, Chitchanok Chuengsatiansup, Tanja Lange, and Christine van Vredendaal. NTRU prime: Reducing attack surface at low cost. In $S A C$ 2017, pages 235-260, 2017.

[BL] Daniel J. Bernstein and Tanja Lange. eBACS: ECRYPT Benchmarking of Cryptographic Systems. https://bench.cr.yp.to, accessed 3 January 2022.

$\left[\mathrm{BGM}^{+} 16\right]$ Andrej Bogdanov, Siyao Guo, Daniel Masny, Silas Richelson, and Alon Rosen. On the hardness of learning with rounding over small modulus. In TCC 2016, pages 209-224, 2016.

[BD20] Zvika Brakerski and Nico Döttling. Lossiness and Entropic Hardness for Ring-LWE. In TCC 2020, 2020.

[BGPW16] Johannes Buchmann, Florian Göpfert, Rachel Player, and Thomas Wunderer. On the hardness of LWE with binary error: Revisiting the hybrid latticereduction and meet-in-the-middle attack. In AFRICACRYPT 2016, pages 24-43, 2016.

[CL21] André Chailloux and Johanna Loyer. Lattice sieving via quantum random walks. In ASIACRYPT 2021, 2021.

$\left[\mathrm{CDH}^{+} 20\right]$ Cong Chen, Oussama Danba, Jeffrey Hoffstein, Andreas Hülsing, Joost Rijneveld, John M. Schanck, Peter Schwabe, William Whyte, and Zhenfei Zhang. NTRU: A submission to the NIST post-quantum standardization effort. 2020. https://ntru.org/. 
[CHWZ17] Cong Chen, Jeffrey Hoffstein, William Whyte, and Zhenfei Zhang. NIST PQ Submission: NTRUEncrypt A lattice based encryption algorithm. 2017. https://ntru.org/resources. shtml.

[CZZ18] Long Chen, Zhenfeng Zhang, and Zhenfei Zhang. On the hardness of the computational Ring-LWR problem and its applications. In ASIACRYPT 2018, pages 435-464, 2018.

[CN11] Yuanmi Chen and Phong Q. Nguyen. BKZ 2.0: Better Lattice Security Estimates. In ASIACRYPT 2011, pages 1-20, 2011.

[CKLS18] Jung Hee Cheon, Duhyeong Kim, Joohee Lee, and Yongsoo Song. Lizard: Cut off the tail! A practical post-quantum public-key encryption from LWE and LWR. In International Conference on Security and Cryptography for Networks, pages 160-177, 2018.

$\left[\mathrm{CPS}^{+} 20\right]$ Chitchanok Chuengsatiansup, Thomas Prest, Damien Stehlé, Alexandre Wallet, and Keita Xagawa. ModFalcon: compact signatures based on module NTRU lattices. In ASIACCS 2020, 2020.

[CHK $\left.{ }^{+} 21\right]$ Chi-Ming Marvin Chung, Vincent Hwang, Matthias J. Kannwischer, Gregor Seiler, Cheng-Jhih Shih, and Bo-Yin Yang. NTT Multiplication for NTT-unfriendly Rings. IACR Transactions on Cryptographic Hardware and Embedded Systems, page to appear, 2021.

[DSDGR20] Dana Dachman-Soled, Léo Ducas, Huijing Gong, and Mélissa Rossi. Lwe with side information: Attacks and concrete security estimation. In Crypto 2020, 2020.

$\left[\mathrm{DHP}^{+} 20\right]$ Dipayan Das, Jeffrey Hoffstein, Jill Pipher, William Whyte, and Zhenfei Zhang. Modular lattice signatures, revisited. Designs, Codes and Cryptography, 88(3):505-532, 2020.

[Den03] Alexander W Dent. A designer's guide to KEMs. In IMA International Conference on Cryptography and Coding, pages 133-151. Springer, 2003.

[DFMS21] Jelle Don, Serge Fehr, Christian Majenz, and Christian Schaffner. Onlineextractability in the quantum random-oracle model. Cryptology ePrint Archive, Report 2021/280, 2021. https://ia.cr/2021/280.

[DDLL13] Léo Ducas, Alain Durmus, Tancrède Lepoint, and Vadim Lyubashevsky. Lattice Signatures and Bimodal Gaussians. In CRYPTO 2013, pages 40-56, 2013.

[DLP14] Léo Ducas, Vadim Lyubashevsky, and Thomas Prest. Efficient Identity-Based Encryption over NTRU Lattices. In ASIACRYPT 2014, pages 22-41, 2014.

[DN12] Léo Ducas and Phong Q. Nguyen. Learning a Zonotope and More: Cryptanalysis of NTRUSign Countermeasures. In ASIACRYPT 2012, pages 433-450, 2012.

[DP16] Léo Ducas and Thomas Prest. Fast Fourier Orthogonalization. In ISSAC 2016, pages 191-198, 2016.

[DHK $\left.{ }^{+} 21\right]$ Julien Duman, Kathrin Hövelmanns, Eike Kiltz, Vadim Lyubashevsky, Gregor Seiler, and Dominique Unruh. A Thorough Treatment of Highly-Efficient NTRU Instantiations. Cryptology ePrint Archive, Report 2021/1352, 2021. https://ia.cr/2021/1352. 
$\left[\mathrm{FHK}^{+} 20\right]$ Pierre-Alain Fouque, Jeffrey Hoffstein, Paul Kirchner, Vadim Lyubashevsky, Thomas Pornin, Thomas Prest, Thomas Ricosset, Gregor Seiler, William Whyte, and Zhenfei Zhang. Falcon: Fast-Fourier Lattice-based Compact Signatures over NTRU. 2020. https://falcon-sign.info/.

[FO99] Eiichiro Fujisaki and Tatsuaki Okamoto. Secure integration of asymmetric and symmetric encryption schemes. In Crypto'99, pages 537-554, 1999.

[GPV08] Craig Gentry, Chris Peikert, and Vinod Vaikuntanathan. Trapdoors for Hard Lattices and New Cryptographic Constructions. In STOC 2008, pages 197-206, 2008.

[GGH97a] Oded Goldreich, Shafi Goldwasser, and Shai Halevi. Public-Key Cryptosystems from Lattice Reduction Problems. In CRYPTO '97, pages 112-131, 1997.

[GGH97b] Oded Goldreich, Shafi Goldwasser, and Shai Halevi. Public-key cryptosystems from lattice reduction problems. In Crypto'97, pages 112-131, 1997.

$\left[\mathrm{HHP}^{+} 03\right]$ Jeffrey Hoffstein, Nick Howgrave-Graham, Jill Pipher, Joseph H. Silverman, and William Whyte. NTRUSIGN: Digital Signatures Using the NTRU Lattice. In $C T-R S A$ 2003, pages 122-140, 2003.

[HPS98] Jeffrey Hoffstein, Jill Pipher, and Joseph H Silverman. NTRU: A ring-based public key cryptosystem. In ANTS 1998, pages 267-288, 1998.

[HHK17] Dennis Hofheinz, Kathrin Hövelmanns, and Eike Kiltz. A modular analysis of the Fujisaki-Okamoto transformation. In TCC 2017, pages 341-371, 2017.

[HG07] Nick Howgrave-Graham. A hybrid lattice-reduction and meet-in-the-middle attack against ntru. In CRYPTO 200\%, pages 150-169, 2007.

[HRSS17] Andreas Hülsing, Joost Rijneveld, John Schanck, and Peter Schwabe. HighSpeed Key Encapsulation from NTRU. In CHES 2017, pages 232-252, 2017.

$\left[\mathrm{JAC}^{+} 20\right]$ David Jao, Reza Azarderakhsh, Matthew Campagna, Craig Costello, Luca De Feo, Basil Hess, Amir Jalali, Brian Koziel, Brian LaMacchia, Patrick Longa, Michael Naehrig, Joost Renes, Vladimir Soukharev, David Urbanik, and Geovandro Pereira. Supersingular Isogeny Key Encapsulation. 2020. https: //sike.org/files/SIDH-spec.pdf.

[KEF20] Paul Kirchner, Thomas Espitau, and Pierre-Alain Fouque. Fast reduction of algebraic lattices over cyclotomic fields. In CRYPTO 2020, pages 155-185, 2020 .

[KF17] Paul Kirchner and Pierre-Alain Fouque. Revisiting lattice attacks on overstretched NTRU parameters. In EUROCRYPT 2017, pages 3-26, 2017.

[LATV12] Adriana López-Alt, Eran Tromer, and Vinod Vaikuntanathan. On-the-fly multiparty computation on the cloud via multikey fully homomorphic encryption. In STOC 2012, pages 1219-1234, 2012.

$\left[\mathrm{LLJ}^{+}\right.$19] Xianhui Lu, Yamin Liu, Dingding Jia, Haiyang Xue, Jingnan He, Zhenfei Zhang, Zhe Liu, Hao Yang, Bao Li, and Kunpeng Wang. LAC: Lattice-based Cryptosystems. 2019. https://csrc.nist.gov/Projects/ Post-Quantum-Cryptography/Round-2-Submissions. 
[LS18] Vadim Lyubashevsky and Gregor Seiler. Short, invertible elements in partially splitting cyclotomic rings and applications to lattice-based zero-knowledge proofs. In EUROCRYPT 2018, pages 204-224, 2018.

[LS19] Vadim Lyubashevsky and Gregor Seiler. NTTRU: Truly Fast NTRU Using NTT. IACR Transactions on Cryptographic Hardware and Embedded Systems, pages 180-201, 2019.

[Mic01] Daniele Micciancio. Improving lattice based cryptosystems using the Hermite normal form. In International Cryptography and Lattices Conference, pages $126-145,2001$.

[Ngu21] Phong Q Nguyen. Boosting the Hybrid Attack on NTRU: Torus LSH, Permuted HNF and Boxed Sphere. 2021.

[NR06] Phong Q. Nguyen and Oded Regev. Learning a Parallelepiped: Cryptanalysis of GGH and NTRU Signatures. In EUROCRYPT 2006, pages 271-288, 2006.

[NIS20] NIST. Round 3 candidates of the NIST Post-Quantum Cryptography Standardization, 2020. https://csrc.nist.gov/Projects/ post-quantum-cryptography/round-3-submissions.

[PST20] Christian Paquin, Douglas Stebila, and Goutam Tamvada. Benchmarking post-quantum cryptography in TLS. In PQCrypto 2020, pages 72-91, 2020.

[Por20] Thomas Pornin. Efficient Elliptic Curve Operations On Microcontrollers With Finite Field Extensions. Cryptology ePrint Archive, Report 2020/009, 2020. https://eprint.iacr.org/2020/009.

[PP19] Thomas Pornin and Thomas Prest. More efficient algorithms for the NTRU key generation using the field norm. In PKC 2019, pages 504-533, 2019.

[Pre15] Thomas Prest. Gaussian Sampling in Lattice-Based Cryptography. PhD thesis, École Normale Supérieure, 2015.

[Saa19] Markku-Juhani O. Saarinen. On PQC message lengths and some energy consumption myths, 2019. https://groups.google.com/a/list.nist.gov/ forum/\#! forum/pqc-forum.

[SA15] Markku-Juhanie Saarinen and Jean-Philippe Aumasson. RFC 7693: The BLAKE2 Cryptographic Hash and Message Authentication Code (MAC), 2015.

[SE94] Claus-Peter Schnorr and Martin Euchner. Lattice basis reduction: Improved practical algorithms and solving subset sum problems. Mathematical programming, 66(1-3):181-199, 1994.

[SKD20a] Dimitrios Sikeridis, Panos Kampanakis, and Michael Devetsikiotis. Assessing the overhead of post-quantum cryptography in TLS 1.3 and SSH. In Dongsu Han and Anja Feldmann, editors, CoNEXT '20: The 16th International Conference on emerging Networking EXperiments and Technologies, Barcelona, Spain, December, 2020, pages 149-156. ACM, 2020.

[SKD20b] Dimitrios Sikeridis, Panos Kampanakis, and Michael Devetsikiotis. Postquantum authentication in TLS 1.3: A performance study. In 27th Annual Network and Distributed System Security Symposium, NDSS 2020, San Diego, California, USA, February 23-26, 2020. The Internet Society, 2020. 
[SC19] Yongha Son and Jung Hee Cheon. Revisiting the hybrid attack on sparse secret LWE and application to HE parameters. In WAHC 2019, pages 11-20, 2019 .

[SS11] Damien Stehlé and Ron Steinfeld. Making NTRU as secure as worst-case problems over ideal lattices. In EUROCRYPT 2011, pages 27-47, 2011.

$\left[\mathrm{ZYF}^{+} 20\right]$ Jiang Zhang, Yu Yu, Shuqin Fan, Zhenfeng Zhang, and Kang Yang. Tweaking the Asymmetry of Asymmetric-Key Cryptography on Lattices: KEMs and Signatures of Smaller Sizes. In PKC 2020, 2020.

\section{A Argument for Claim 1}

To argue Claim 1, we need Claim 4 to estimate the norm of the involved vectors.

Claim 4. Let $\mathcal{R}=\mathbb{Z}[x] /\left(x^{n}+1\right)$ with $n$ a power-of-2. Let $f, g \in \mathcal{R}$ be iid-random over $D_{\mathbb{Z}, \sigma_{f}}, \mathbf{B}_{f, g}=\left(\begin{array}{ll}g & G \\ f & F\end{array}\right) \in \mathcal{R}^{2 \times 2}$ be an NTRU basis. Let $v=\frac{\gamma^{2} F \bar{f}+G \bar{g}}{\gamma^{2} f \bar{f}+g \bar{g}}$ with $\gamma>0$. Let $\left(G^{\perp}, F^{\perp}\right)=(G-g v, \gamma F-\gamma f v)$ and $\left(G^{\prime}, F^{\prime}\right)=\left(G-g\lfloor v\rceil_{q^{\prime}}, F-f\lfloor v\rceil_{q^{\prime}}\right)$. Let $I(\gamma)=\frac{2 \ln (\gamma)}{\gamma^{2}-1}$ for $\gamma \neq 1$ and $I(1)=1$. Then

- $\left\|\left(G^{\perp}, F^{\perp}\right)\right\| \approx \frac{q \gamma}{\sqrt{n} \cdot \sigma_{f}} \sqrt{I(\gamma)}$;

- $\left\|\left(G^{\prime}, \gamma F^{\prime}\right)\right\| \leq \frac{q \gamma}{\sqrt{n} \cdot \sigma_{f}} \sqrt{I(\gamma)}+\frac{n^{1.5} \sqrt{\gamma^{2}+1}}{2 q^{\prime}} \sigma_{f}$.

Argument. First, it can be verified that $\left(G^{\perp}, F^{\perp}\right)=q \gamma\left(\frac{-\gamma \bar{f}}{\gamma^{2} f \bar{f}+g \bar{g}}, \frac{\bar{g}}{\gamma^{2} f \bar{f}+g \bar{g}}\right)$. Let $\xi_{n}$ be a $2 n$-th primitive root of 1 , then $\|f\|^{2}=\frac{1}{n} \sum_{i=1}^{n} f\left(\xi_{n}^{2 i-1}\right) \bar{f}\left(\xi_{n}^{2 i-1}\right)$ for any $f \in \mathbb{K}_{n}$. Some routine computation yields that

$$
\left\|\left(G^{\perp}, F^{\perp}\right)\right\|^{2}=\frac{q^{2} \gamma^{2}}{n} \sum_{i=1}^{n} \frac{1}{\gamma^{2} f\left(\xi_{n}^{2 i-1}\right) \bar{f}\left(\xi_{n}^{2 i-1}\right)+g\left(\xi_{n}^{2 i-1}\right) \bar{g}\left(\xi_{n}^{2 i-1}\right)} .
$$

We heuristically model all these $f\left(\xi_{n}^{2 i-1}\right) \bar{f}\left(\xi_{n}^{2 i-1}\right)$ and $g\left(\xi_{n}^{2 i-1}\right) \bar{g}\left(\xi_{n}^{2 i-1}\right)$ as independent random variables drawn from the chi-square distribution of parameter $k=2$ scaled by $\frac{n}{2} \sigma_{f}^{2}$. The average of $\frac{\frac{n}{2} \sigma_{f}^{2}}{q^{2} \gamma^{2}} \cdot\left\|\left(G^{\perp}, F^{\perp}\right)\right\|^{2}$ is then estimated as

$$
\int_{0}^{\infty} \int_{0}^{\infty} \frac{1}{\gamma^{2} x+y} \frac{e^{-\frac{x+y}{2}}}{4} d x d y=\int_{0}^{\infty} \int_{0}^{z} \frac{1}{\left(\gamma^{2}-1\right) x+z} \frac{e^{-\frac{z}{2}}}{4} d x d z=\frac{I(\gamma)}{2}
$$

Further, we get the following approximation $\left\|\left(G^{\perp}, F^{\perp}\right)\right\| \approx \frac{q \gamma}{\sqrt{n} \cdot \sigma_{f}} \sqrt{I(\gamma)}$.

Let $\Delta v=v-\lfloor v\rceil_{q^{\prime}}$, then $\|\Delta v\|_{\infty} \leq \frac{1}{2 q^{\prime}}$. It is known that $\|f \Delta v\| \leq \frac{n}{2 q^{\prime}}\|f\|$ for any $f \in \mathbb{K}_{n}$. We estimate $\|f\|$ and $\|g\|$ as $\sqrt{n} \sigma_{f}$. Then it follows that

$$
\begin{aligned}
\left\|\left(G^{\prime}, \gamma F^{\prime}\right)\right\| & \leq\left\|\left(G^{\perp}, F^{\perp}\right)\right\|+\|(g \Delta v, \gamma f \Delta v)\| \\
& \leq\left\|\left(G^{\perp}, F^{\perp}\right)\right\|+\frac{n}{2 q^{\prime}}\|(g, \gamma f)\| \\
& \approx \frac{q \gamma}{\sqrt{n} \cdot \sigma_{f}} \sqrt{I(\gamma)}+\frac{n^{1.5} \sqrt{\gamma^{2}+1}}{2 q^{\prime}} \sigma_{f} .
\end{aligned}
$$

This completes the argument. 
Remark 5. When $\gamma=1$, our estimation of $\left\|\left(G^{\perp}, F^{\perp}\right)\right\|$ is $\frac{q}{\sqrt{n} \cdot \sigma_{f}}$. This implies that for optimal trapdoor, $\sigma_{f}$ should be $\sqrt[4]{2} \sqrt{\frac{q}{2 n}} \approx 1.19 \sqrt{\frac{q}{2 n}}$ slightly larger than the previous heuristic bound $1.17 \sqrt{\frac{q}{2 n}}$ suggested in [DLP14]. The explanation of the factor 1.17 is given in [Pre15]: the argument makes use of the projection heuristic on both $(g, f)$ and $(G, F)$ two parts. In fact, this explanation seems problematic: as $\mathbf{B}_{f, g}$ is symplectic, once the Gram-Schmidt norms of the $(f, g)$ part are fixed, those of the $(G, F)$ part are determined as well. Thus, the optimal bound for the Gram-Schmidt norm of $\mathbf{B}_{f, g}$ is entirely determined by $\frac{\left\|\mathbf{b}_{0}^{*}\right\|^{2}}{\left\|\mathbf{b}_{n-1}^{*}\right\|^{2}}=\operatorname{Tr}(f \bar{f}+g \bar{g}) \operatorname{Tr}\left(\frac{1}{f \bar{f}+g \bar{g}}\right)$ where $\operatorname{Tr}$ denotes the trace over $\mathbb{K}_{n}$.

Argument of Claim 1. Let $s^{\prime}=s-\mu_{s} \mathbf{1}$ and $e^{\prime}=e-\mu_{e} \mathbf{1}$. It can be verified that

$$
c^{\prime}=\left(Q\left(g s^{\prime}+f e^{\prime}\right) \bmod q Q\right) \text { and } c^{\prime \prime}=\left(q^{\prime} Q\left(F e^{\prime}+G s^{\prime}\right)-c^{\prime} w \bmod q q^{\prime} Q\right) .
$$

If $\left\|f e^{\prime}+g s^{\prime}\right\|_{\infty} \leq \frac{q}{2}$, then $c^{\prime}=Q\left(g s^{\prime}+f e^{\prime}\right)$ and $c^{\prime \prime}=\left(Q\left(F_{d} e^{\prime}+G_{d} s^{\prime}\right) \bmod q q^{\prime} Q\right)$. Further, if $\max \left\{\left\|f e^{\prime}+g s^{\prime}\right\|_{\infty}, \frac{\left\|F_{d} e^{\prime}+G_{d} s^{\prime}\right\|_{\infty}}{q^{\prime}}\right\} \leq \frac{q}{2}$, it follows that $c^{\prime}=Q\left(g s^{\prime}+f e^{\prime}\right)$ and $c^{\prime \prime}=Q\left(F_{d} e^{\prime}+G_{d} s^{\prime}\right)$, which ensures a correct decoding similar to the RO algorithm.

Let $T_{1}=f e^{\prime}+g s^{\prime}$ and $T_{2}=\frac{F_{d}}{q^{\prime}} e^{\prime}+\frac{G_{d}}{q^{\prime}} s^{\prime}$. We approximate $T_{1}, T_{2}$ by two random Gaussian vectors of standard deviations $\sigma_{T_{1}}=\sqrt{n} \sigma_{f} \sigma_{s} \sqrt{\gamma^{2}+1}=\sigma_{1}$ and $\sigma_{T_{2}}=\frac{\sigma_{s}}{q^{\prime}}\left\|\left(G_{d}, \gamma F_{d}\right)\right\|$. By Claim 4, we have $\sigma_{T_{2}} \leq \sigma_{2}$ and then $\tau \leq \min \left\{\frac{q}{2 \sqrt{2} \sigma_{T_{1}}}, \frac{q}{2 \sqrt{2} \sigma_{T_{2}}}\right\}$. Therefore, the probability of correct decoding is at least $1-2 n \cdot(1-\operatorname{erf}(\tau))$. 